\title{
Asymmetric Nickel-Catalyzed Negishi Cross-Couplings of Secondary $\alpha$-Bromo Amides with Organozinc Reagents
}

\author{
Christian Fischer and Gregory C. Fu* \\ Department of Chemistry, Massachusetts Institute of Technology, \\ Cambridge, Massachusetts 02139
}

\section{Supporting Information}

\section{General}

All reactions were carried out in oven-dried glassware under an atmosphere of nitrogen or argon. 1,3-Dimethyl-2-imidazolidinone (DMI) was purchased from Fluka (anhydrous), and THF was purified as described by Grubbs. ${ }^{1} \mathrm{NiCl}_{2} \cdot$ glyme (Strem) and $(S)$ - and (R)-(i-Pr)-Pybox (Aldrich) were used as received. All other chemicals were purchased and used without further purification, unless noted otherwise.

Note: Although $\mathrm{NiCl}_{2} \cdot$ glyme and ( $i$-Pr)-Pybox can be left in air without any impact on cross-coupling efficiency, we recommend that, for long-term storage, they be kept under an inert atmosphere.

Chromatographic purification was performed using Sorbent silica 32-63, $60 \AA$. TLC was performed on Merck silica gel $60 \mathrm{~F}_{254}$ TLC glass plates and visualized with ultraviolet light or permanganate stain.

Melting points were measured on a Hoover melting point apparatus using open glass capillaries, and the values are uncorrected. Infrared spectra were recorded on a Perkin Elmer Spectrum 2000 FTIR spectrophotometer as thin films. Optical rotations were measured on a JASCO P-1010 digital polarimeter in $10 \mathrm{~cm}, 1.0 \mathrm{~mL}$ cells. The concentration in $\mathrm{g} / 100 \mathrm{~mL}$ and the solvent are given in parentheses. High resolution mass spectrometric measurements were performed by the mass spectrometry service of the DCIF at MIT on a Bruker Daltonics APEXII 3 Tesla Fourier Transform Mass Spectrometer system.

HPLC analyses were carried out on an Agilent 1100 series system with Daicel Chiralpak ${ }^{\circledR}$ columns in hexanes/iso-propanol mixtures. GC analysis (achiral) was performed on a Hewlett-Packard HP 6890 series apparatus on a DB-5 column. GC analysis (chiral) was performed on a Hewlett-Packard HP 6850 series apparatus on a Chrompack Capillary Column 'CP-Chirasil-Dex CB' 25 m 0.25 mm 0.25 um \#CP7502, unless otherwise noted.

${ }^{1} \mathrm{H}$ NMR spectra were recorded on a VARIAN Inova $500 \mathrm{MHz}$ spectrometer in chloroform-d. All signals are reported in ppm with the internal chloroform signal at $7.26 \mathrm{ppm}$ as the standard. The data are reported as: $(\mathrm{s}=$ singlet, $\mathrm{d}=$ doublet, $\mathrm{t}=$ triplet, $\mathrm{q}=$ quartet, $\mathrm{m}=$ multiplet or unresolved, $\mathrm{br}=$ broad signal, coupling constant(s) in $\mathrm{Hz}$, integration). ${ }^{13} \mathrm{C}$ NMR spectra were recorded with ${ }^{1} \mathrm{H}$-decoupling on a VARIAN Inova $500 \mathrm{MHz}$ spectrometer in chloroform-d. All signals are reported in ppm with the internal chloroform signal at $77.0 \mathrm{ppm}$ as the standard. 


\section{Preparation of Materials}

Synthesis of $\boldsymbol{\alpha}$-bromo amides: representative procedure. A 100-mL flask was charged with $\mathrm{N}$-benzylaniline $(5.04 \mathrm{~g}, 27.5 \mathrm{mmol})$, evacuated, and back-filled with argon. The flask was cooled to $0^{\circ} \mathrm{C}$ in an ice bath, and NEt $3(3.83 \mathrm{~mL}, 27.5 \mathrm{mmol})$ and THF (50 mL) were added. The orange solution was stirred for 5 minutes, and then the $\alpha$-bromo acid bromide ( $25 \mathrm{mmol}$ ) was added slowly via syringe, leading to the formation of a precipitate. The resulting suspension was stirred for 10 minutes at $0{ }^{\circ} \mathrm{C}$, and then the reaction was quenched by the addition of ice/water. The mixture was extracted three times with $\mathrm{Et}_{2} \mathrm{O}(50 \mathrm{~mL})$, and then the combined extracts were washed with brine, dried over sodium sulfate, and concentrated. The resulting oil was purified by flash chromatography (hexanes/ethyl acetate), which furnished the $\alpha$-bromo amide.

( \pm )- $N$-Benzyl- $N$-phenyl-2-bromobutanamide

mp: $37-39{ }^{\circ} \mathrm{C}$;

${ }^{1} \mathrm{H} \mathrm{NMR}\left(\mathrm{CDCl}_{3}, 500 \mathrm{MHz}\right) \delta$ 7.37-7.34 (m, 3H), 7.30-7.26 (m, 3H), 7.24-7.20 (m, 2H), $7.05(\mathrm{br} \mathrm{s}, 2 \mathrm{H}), 4.94(\mathrm{~d}, J=14.0 \mathrm{~Hz}, 1 \mathrm{H}), 4.88(\mathrm{~d}, J=14.0 \mathrm{~Hz}, 1 \mathrm{H}), 3.98(\mathrm{t}, J=6.7 \mathrm{~Hz}, 1 \mathrm{H})$, $2.21(\mathrm{dq}, J=6.7,7.3 \mathrm{~Hz}, 1 \mathrm{H}), 1.97(\mathrm{dq}, 6.7,7.3 \mathrm{~Hz}, 1 \mathrm{H}), 0.91(\mathrm{t}, J=7.3 \mathrm{~Hz}, 3 \mathrm{H})$;

${ }^{13} \mathrm{C}$ NMR $\left(\mathrm{CDCl}_{3}, 125 \mathrm{MHz}\right) \delta 169.0,141.0,136.8,129.7,128.8,128.5,128.4,128.3$, $127.5,53.5,45.9,28.6,12.1$;

IR (film) 1667, 1495, 1404, $668 \mathrm{~cm}^{-1}$;

HRMS (ESI) calcd for $\mathrm{C}_{17} \mathrm{H}_{18} \mathrm{BrNONa}\left(\mathrm{M}+\mathrm{Na}^{+}\right)$354.0464, found 354.0461.

\section{( \pm )- $N$-Benzyl- $N$-phenyl-2-bromohexanamide}

${ }^{1} \mathrm{H}$ NMR $\left(\mathrm{CDCl}_{3}, 500 \mathrm{MHz}\right)$ \& 7.36-7.34 (m, 3H), 7.29-7.25 (m, 3H), 7.22-7.20 (m, 2H), $7.06(\mathrm{br} \mathrm{s}, 2 \mathrm{H}), 4.96(\mathrm{~d}, J=14.0 \mathrm{~Hz}, 1 \mathrm{H}), 4.86(\mathrm{~d}, J=14.3 \mathrm{~Hz}, 1 \mathrm{H}), 4.04(\mathrm{dd}, J=6.7,7.2 \mathrm{~Hz}$, $1 \mathrm{H}), 2.23-2.16(\mathrm{~m}, 1 \mathrm{H}), 1.98-1.93(\mathrm{~m}, 1 \mathrm{H}), 1.30-1.21(\mathrm{~m}, 4 \mathrm{H}), 0.88-0.85(\mathrm{~m}, 3 \mathrm{H})$;

${ }^{13} \mathrm{C} \mathrm{NMR}\left(\mathrm{CDCl}_{3}, 125 \mathrm{MHz}\right) \delta 169.1,141.0,136.8,129.6,128.8,128.5,128.4,128.2$, 127.5, 53.5, 44.4, 35.0, 29.5, 22.1, 13.8;

IR (film) 2958, 2930, 1667, 1595, $1403 \mathrm{~cm}^{-1}$;

HRMS (ESI) calcd for $\mathrm{C}_{19} \mathrm{H}_{22} \mathrm{BrNONa}\left(\mathrm{M}+\mathrm{Na}^{+}\right) 382.0782$, found 382.0782.

\section{( \pm - $\mathrm{N}$-Benzyl- $\mathrm{N}$-phenyl-2-bromo-4-methylpentanamide}

${ }^{1} \mathrm{H} \mathrm{NMR}\left(\mathrm{CDCl}_{3}, 500 \mathrm{MHz}\right)$ \& 7.36-7.35 (m, 3H), 7.30-7.26 (m, 3H), 7.22-7.20 (m, 2H), $7.07(\mathrm{br} \mathrm{s}, 2 \mathrm{H}), 4.97(\mathrm{~d}, J=14.0 \mathrm{~Hz}, 1 \mathrm{H}), 4.84(\mathrm{~d}, J=14.0 \mathrm{~Hz}, 1 \mathrm{H}), 4.12(\mathrm{dd}, J=7.3,7.6 \mathrm{~Hz}$, $1 \mathrm{H}), 2.09-2.03(\mathrm{~m}, 1 \mathrm{H}), 1.89-1.83(\mathrm{~m}, 1 \mathrm{H}), 1.67-1.61(\mathrm{~m}, 1 \mathrm{H}), 0.81(\mathrm{~d}, J=6.7 \mathrm{~Hz}, 3 \mathrm{H}), 0.76$ $(\mathrm{d}, J=6.4 \mathrm{~Hz}, 3 \mathrm{H})$;

${ }^{13} \mathrm{C} \mathrm{NMR}\left(\mathrm{CDCl}_{3}, 125 \mathrm{MHz}\right) \delta 169.1,141.0,136.8,129.6,128.8,128.6,128.4,128.2$, $127.5,55.5,44.0,43.4,26.3,22.2,22.1$;

IR (film) 2957, 1667, 1595, 1494, $1402 \mathrm{~cm}^{-1}$;

HRMS (ESI) calcd for $\mathrm{C}_{19} \mathrm{H}_{23} \mathrm{BrNO}\left(\mathrm{M}+\mathrm{H}^{+}\right) 360.0958$, found 360.0969 .

( \pm )-N-Benzyl-N-phenyl-2-bromopropanamide mp: $61-62{ }^{\circ} \mathrm{C}$; 
${ }^{1} \mathrm{H} \mathrm{NMR}\left(\mathrm{CDCl}_{3}, 500 \mathrm{MHz}\right)$ 8 7.37-7.34 (m, 3H), 7.30-7.26 (m, 3H), 7.21-7.19 (m, 2H), $7.07(\mathrm{br} \mathrm{s}, 2 \mathrm{H}), 4.99(\mathrm{~d}, J=14.3 \mathrm{~Hz}, 1 \mathrm{H}), 4.80(\mathrm{~d}, J=14.3 \mathrm{~Hz}, 1 \mathrm{H}), 4.24(\mathrm{q}, J=6.7 \mathrm{~Hz}, 1 \mathrm{H})$, $1.77(\mathrm{~d}, J=6.7 \mathrm{~Hz}, 3 \mathrm{H})$;

${ }^{13} \mathrm{C} \mathrm{NMR}\left(\mathrm{CDCl}_{3}, 125 \mathrm{MHz}\right) \delta 169.6,141.0,136.8,129.6,128.8,128.6,128.5,128.2$, $127.5,53.6,39.4,21.8$; IR (film) 1667, 1595, 1495, 1447, 1402, 1259, $1180 \mathrm{~cm}^{-1}$;

HRMS (ESI) calcd for $\mathrm{C}_{16} \mathrm{H}_{16} \mathrm{BrNONa}\left(\mathrm{M}+\mathrm{Na}^{+}\right)$340.0307, found 340.0324.

Preparation of the organozinc reagents: general procedure. ${ }^{2}$ A $25-\mathrm{mL}$ Schlenk flask was charged with zinc powder $(0.98 \mathrm{~g}, 15 \mathrm{mmol})$ and heated to $70{ }^{\circ} \mathrm{C}$ under high vacuum for 30 minutes. After back-filling with argon, DMI (to give a total volume of 10 $\mathrm{mL})$ and iodine $(0.13 \mathrm{~g}, 0.50 \mathrm{mmol})$ were added. After the red color of iodine had faded, the alkyl halide ( $10 \mathrm{mmol}$; freshly distilled) was added. The colorless reaction mixture was stirred for $12 \mathrm{~h}$ at $70{ }^{\circ} \mathrm{C}$ (Exceptions: For MeI, the reaction was not heated; for cyclohexylmethylbromide, the reaction was heated at $85^{\circ} \mathrm{C}$ for $24 \mathrm{~h}$ ), and then it was cooled to room temperature (The disappearance of the starting material and the formation of the organozinc reagent can readily be monitored by no-D NMR). The gray solution $(\sim 1.0 \mathrm{M})$ was transferred into a dry vessel via cannula.

These organozinc solutions can be stored at room temperature for several weeks in a dry atmosphere without deterioration. 


\section{Negishi Cross-Coupling Reactions}

Eq 2. In a glove box, a 4-mL vial was charged with $\mathrm{Ni}(\operatorname{cod})_{2}(2.8 \mathrm{mg}, 10 \mu \mathrm{mol}),(S)$ $(s-B u)$-Pybox $(6.6 \mathrm{mg}, 20 \mu \mathrm{mol})$, and DMA $(0.60 \mathrm{~mL})$. The dark-blue solution was stirred for 20 minutes, and then ( \pm )-2-bromo- $N, N$-diethylpropanamide $(52 \mathrm{mg}, 0.25 \mathrm{mmol}$ ) was added. The vial was closed with a septum cap, removed from the glove box, and fitted with an argon inlet. Then, HexZnBr ( 1.0 M in DMA; $0.40 \mathrm{~mL}, 0.40 \mathrm{mmol})$ was added. The resulting dark-brown reaction mixture was stirred for $13 \mathrm{~h}$ at r.t. Then, the excess organozinc reagent was quenched by the addition of ethanol $(0.5 \mathrm{~mL})$, and $n$ decane (calibrated internal standard; $48.8 \mu \mathrm{L}, 0.25 \mathrm{mmol}$ ) was added. The brown mixture was passed through a plug of silica gel (eluted with $\mathrm{Et}_{2} \mathrm{O}$ ) to remove inorganic salts and most of the DMI. The filtrate was analyzed by GC to determine the conversion. The enantiomeric excess was determined on chiral GC [GTA column, $\mathrm{t}_{\mathrm{r}}$ (major) $33.0 \mathrm{~min}, \mathrm{t}_{\mathrm{r}}$ (minor) $34.0 \mathrm{~min}$; heating program: $80{ }^{\circ} \mathrm{C} \rightarrow 120^{\circ} \mathrm{C} @ 1.0$ ${ }^{\circ} \mathrm{C} / \mathrm{min}$ ]. Run $1: 5 \% \mathrm{~N}, \mathrm{~N}$-diethyl-2-methyloctanamide (20\% ee); Run 2: $4 \% \mathrm{~N}, \mathrm{~N}$-diethyl2-methyloctanamide (18\% ee).

Eq 3. In a glove box, a 4-mL vial was charged with $\mathrm{Ni}(\operatorname{cod})_{2}(2.8 \mathrm{mg}, 10 \mu \mathrm{mol}),(S)$ $(s-B u)$-Pybox $(6.6 \mathrm{mg}, 20 \mu \mathrm{mol})$, and DMA $(0.60 \mathrm{~mL})$. The dark-blue solution was stirred for 20 minutes, and then ( \pm - $N$-benzyl- $N$-phenyl-2-bromobutanamide $(83 \mathrm{mg}, 0.25$ $\mathrm{mmol}$ ) was added. The vial was closed with a septum cap, removed from the glove box, and fitted with an argon inlet. Then, $\operatorname{HexZnBr}(\sim 1.0 \mathrm{M}$ in DMA; $0.40 \mathrm{~mL}, 0.40$ $\mathrm{mmol}$ ) was added. The resulting dark-brown reaction mixture was stirred for $13 \mathrm{~h}$ at r.t. Then, the excess organozinc reagent was quenched by the addition of ethanol ( 0.5 $\mathrm{mL}$ ), and $n$-decane (calibrated internal standard; $48.8 \mu \mathrm{L}, 0.25 \mathrm{mmol}$ ) was added. The brown mixture was passed through a plug of silica gel (eluted with $\mathrm{Et}_{2} \mathrm{O}$ ) to remove inorganic salts and most of the DMI. The filtrate was analyzed by GC to determine the conversion. The enantiomeric excess was determined on a Daicel Chiralpak ${ }^{\oplus} \mathrm{AD}-\mathrm{H}$ column in hexanes/iso-propanol (99:1, flow $1.0 \mathrm{~mL} / \mathrm{min}$ ) after filtration through silica gel with 10\% iso-propanol in hexanes. Run 1: 22\% (+)-N-benzyl- $N$-phenyl-2ethyloctanamide (HPLC: hexanes/iso-propanol 99:1, flow $1.0 \mathrm{~mL} / \mathrm{min}, 91 \%$ ee); Run 2: 24\% (+)-N-benzyl- $N$-phenyl-2-ethyloctanamide (HPLC: hexanes/iso-propanol 99:1, flow $1.0 \mathrm{~mL} / \mathrm{min}, 91 \%$ ee).

Eq 4. In a glove box, a 4-mL vial was charged with $\mathrm{NiCl}_{2} \cdot$ glyme $(5.5 \mathrm{mg}, 25 \mu \mathrm{mol})$, (R)-(i-Pr)-Pybox (9.8 mg, $33 \mu \mathrm{mol}),( \pm)-N$-benzyl- $N$-phenyl-2-bromobutanamide $(83 \mathrm{mg}$, $0.25 \mathrm{mmol})$, DMI $(0.55 \mathrm{~mL})$, and THF $(0.12 \mathrm{~mL})$. The resulting orange solution was stirred for 20 minutes, and then the vial was closed with a septum cap, removed from the glove box, fitted with an argon inlet, and placed in a $0{ }^{\circ} \mathrm{C}$ bath. The mixture was stirred for 10 minutes, and then HexZnBr ( 1.0 M in DMI; $0.33 \mathrm{~mL}, 0.33 \mathrm{mmol})$ was added. The resulting dark-brown reaction mixture was stirred for $13 \mathrm{~h}$ at $0{ }^{\circ} \mathrm{C}$. Then, the excess organozinc reagent was quenched by the addition of ethanol $(0.5 \mathrm{~mL})$, and $n$ decane (calibrated internal standard; $48.8 \mu \mathrm{L}, 0.25 \mathrm{mmol}$ ) was added. The brown mixture was passed through a plug of silica gel (eluted with $\mathrm{Et}_{2} \mathrm{O}$ ) to remove inorganic salts and most of the DMI. The filtrate was analyzed by GC to determine the conversion. The enantiomeric excess was determined on a Daicel Chiralpak ${ }^{\circledR} \mathrm{AD}-\mathrm{H}$ 
column in hexanes/iso-propanol (99:1, flow $1.0 \mathrm{~mL} / \mathrm{min}$ ) after filtration through silica gel with 10\% iso-propanol in hexanes. Run 1: 90\% (-)- $N$-benzyl- $N$-phenyl-2ethyloctanamide (HPLC: hexanes/iso-propanol 99:1, flow $1.0 \mathrm{~mL} / \mathrm{min}, 96 \%$ ee); Run 2: 88\% (-)-N-benzyl- $N$-phenyl-2-ethyloctanamide (HPLC: hexanes/iso-propanol 99:1, flow $1.0 \mathrm{~mL} / \mathrm{min}, 96 \%$ ee); Run 3: 90\% (-)- $\mathrm{N}$-benzyl- $\mathrm{N}$-phenyl-2-ethyloctanamide (HPLC: hexanes/iso-propanol 99:1, flow $1.0 \mathrm{~mL} / \mathrm{min}, 96 \%$ ee).

General Procedure for Table 1. In the air (no special precautions are necessary), a 10 -mL Schlenk flask was charged with $\mathrm{NiCl}_{2} \cdot$ glyme $(22.0 \mathrm{mg}, 0.100 \mathrm{mmol}),(R)-(i-\mathrm{Pr})-$ Pybox (39.2 $\mathrm{mg}, 0.130 \mathrm{mmol})$, and the $\alpha$-bromo amide $(1.00 \mathrm{mmol})$. The flask was purged with argon for 5 minutes, and then DMI $(2.2 \mathrm{~mL})$ and THF $(0.5 \mathrm{~mL})$ were added. The resulting orange solution was stirred at room temperature for 20 minutes, and then the flask was placed into a $0^{\circ} \mathrm{C}$ bath. The reaction mixture was stirred for 10 minutes, and then the organozinc reagent (1.0 M in DMI; $1.3 \mathrm{~mL}, 1.3 \mathrm{mmol})$ was added. The resulting dark-brown reaction mixture was stirred for $12 \mathrm{~h}$ at $0{ }^{\circ} \mathrm{C}$. Then, the excess organozinc reagent was quenched by the addition of ethanol $(0.5 \mathrm{~mL})$, and the brown mixture was passed through a plug of silica gel (eluted with $\mathrm{Et}_{2} \mathrm{O}$ ) to remove inorganic salts and most of the DMI. The filtrate was concentrated, and the resulting orange oil was purified by flash chromatography.

The ee's were determined on Daicel Chiralpak ${ }^{\circledR}$ columns.

The second runs were conducted with $(S)-(i-\operatorname{Pr})-$ Pybox.

(S)-(-)-N-Benzyl- $N$-phenyl-2-ethyloctanamide (Table 1 , entry 1$)$. The compound was prepared according to the General Procedure with ( \pm )- $N$-benzyl- $N$-phenyl-2bromobutanamide (332 mg, $1.00 \mathrm{mmol}$ ) and HexZnBr ( 1.0 M in DMI; $1.3 \mathrm{~mL}, 1.3$ $\mathrm{mmol}$ ). After chromatography on silica gel (hexanes/ethyl acetate 15:1), the title compound was isolated as a colorless oil: run 1: $303 \mathrm{mg}$ (90\%; 95\% ee); run 2: $305 \mathrm{mg}$ (90\%; 96\% ee). The ee was determined on an AD-H column (hexanes/iso-propanol 99:1, flow $1.0 \mathrm{~mL} / \mathrm{min}$ ) with $\mathrm{t}_{\mathrm{r}}$ (major) $11.6 \mathrm{~min}, \mathrm{t}_{\mathrm{r}}$ (minor) $13.0 \mathrm{~min}$.

$[\alpha]_{D}^{23}=-30.1\left(\mathrm{c}=1.00, \mathrm{CHCl}_{3}\right)$;

${ }^{1} \mathrm{H}$ NMR $\left(\mathrm{CDCl}_{3}, 500 \mathrm{MHz}\right)$ 8 7.34-7.19 (m, 8H), 6.94-6.91 (m, 2H), $4.94(\mathrm{~d}, J=14.3 \mathrm{~Hz}$, $1 \mathrm{H}), 4.89(\mathrm{~d}, J=14.3 \mathrm{~Hz}, 1 \mathrm{H}), 2.20-2.14(\mathrm{~m}, 1 \mathrm{H}), 1.68-1.59(\mathrm{~m}, 2 \mathrm{H}), 1.42-1.10(\mathrm{~m}, 10 \mathrm{H})$, $0.87(\mathrm{t}, J=7.0 \mathrm{~Hz}, 3 \mathrm{H}), 0.81(\mathrm{t}, J=7.5 \mathrm{~Hz}, 3 \mathrm{H})$;

${ }^{13} \mathrm{C} \mathrm{NMR}\left(\mathrm{CDCl}_{3}, 125 \mathrm{MHz}\right) \delta 176.1,142.3,137.9,129.3,129.0,129.0,128.3,127.7$, $127.2,52.9,43.7,33.1,31.7,29.4,27.5,26.2,22.6,14.1,12.2$;

IR (film) 2959, 2927, 2872, 2856, 1654, 1595, 1495, 1455, 1401, 1270, 1241, $1201 \mathrm{~cm}^{-1}$; HRMS (ESI) calcd for $\mathrm{C}_{23} \mathrm{H}_{31} \mathrm{NONa}\left(\mathrm{M}+\mathrm{Na}^{+}\right) 360.2298$, found 360.2293 .

The absolute configuration was assigned by reduction to the corresponding alcohol (eq 7) and comparison of the optical rotation of the alcohol with the literature (see below).

(R)-(-)-N-Benzyl-N-phenyl-2-methylbutanamide (Table 1, entry 2). The compound was prepared according to the General Procedure with ( \pm )- $\mathrm{N}$-benzyl- $\mathrm{N}$-phenyl-2bromobutanamide (332 mg, $1.00 \mathrm{mmol}$ ) and MeZnI ( 1.0 M in DMI; $1.3 \mathrm{~mL}, 1.3 \mathrm{mmol})$. After chromatography on silica gel (hexanes/ethyl acetate 15:1), the title compound was isolated as a colorless oil: run 1: $236 \mathrm{mg}$ (88\%; 91\% ee); run 2: $242 \mathrm{mg}(91 \%$; $90 \%$ ee). 
The ee was determined on an AS-H column (hexanes/iso-propanol 99:1, flow 1.0 $\mathrm{mL} / \mathrm{min}$ ) with $\mathrm{t}_{\mathrm{r}}$ (major) $7.1 \mathrm{~min}, \mathrm{t}_{\mathrm{r}}$ (minor) $7.5 \mathrm{~min}$.

$[\alpha]_{D}^{22}=-45.4\left(\mathrm{c}=1.05, \mathrm{CHCl}_{3}\right)$;

${ }^{1} \mathrm{H}$ NMR $\left(\mathrm{CDCl}_{3}, 500 \mathrm{MHz}\right)$ 8 7.34-7.19 (m, 8H), 6.96-6.93 (m, 2H), $4.96(\mathrm{~d}, J=14.0 \mathrm{~Hz}$, $1 \mathrm{H}), 4.83(\mathrm{~d}, J=14.0 \mathrm{~Hz}, 1 \mathrm{H}), 2.29-2.22(\mathrm{~m}, 1 \mathrm{H}), 1.77-1.68(\mathrm{~m}, 1 \mathrm{H}), 1.35-1.27(\mathrm{~m}, 1 \mathrm{H}), 1.04$ $(\mathrm{d}, J=7.0 \mathrm{~Hz}, 3 \mathrm{H}), 0.81(\mathrm{t}, J=7.3 \mathrm{~Hz}, 3 \mathrm{H})$;

${ }^{13} \mathrm{C} \mathrm{NMR}\left(\mathrm{CDCl}_{3}, 125 \mathrm{MHz}\right) \delta 176.6,142.4,137.8,129.4,128.8,128.5,128.3,127.8$, $127.2,52.8,38.3,27.5,18.0,12.1$;

IR (film) 2964, 1659, 1595, 1495, 1461, 1455, 1400, 1267, 1244, $1202 \mathrm{~cm}^{-1}$;

HRMS (ESI) calcd for $\mathrm{C}_{18} \mathrm{H}_{21} \mathrm{NONa}\left(\mathrm{M}+\mathrm{Na}^{+}\right)$290.1515, found 290.1526 .

The absolute configuration was determined by reduction with LAH and comparison of the resulting alcohol to the authentic racemic and enantiopure [(S)-2-methyl-butanol was purchased from Lancaster] material by chiral GC [ee of the alcohol: 91\%; CPChirasil-Dex CB, $\mathrm{t}_{\mathrm{r}}$ (major) $30.5 \mathrm{~min}, \mathrm{t}_{\mathrm{r}}$ (minor) $31.4 \mathrm{~min}$; heating program: $40{ }^{\circ} \mathrm{C} \rightarrow 65^{\circ} \mathrm{C}$ $\left.@ 0.5^{\circ} \mathrm{C} / \mathrm{min}\right]$ Note: the sense of induction did not switch compared to entry 1--the change in the CIP assignment is due to a change in CIP priorities.

(-)-N-Benzyl- $N$-phenyl-2-ethyl-5-phenylpentanamide (Table 1, entry 3). The compound was prepared according to the General Procedure with $( \pm)-\mathrm{N}$-benzyl- $\mathrm{N}$ phenyl-2-bromobutanamide $(332 \mathrm{mg}, 1.00 \mathrm{mmol})$ and the organozinc reagent $(\sim 1.0 \mathrm{M}$ in DMI; $1.3 \mathrm{~mL}, 1.3 \mathrm{mmol}$ ). After chromatography on silica gel (hexanes/ethyl acetate 10:1), the title compound was isolated as colorless crystals: run 1: $307 \mathrm{mg}$ (83\%; $96 \%$ ee); run 2: $315 \mathrm{mg}(85 \%$; $96 \%$ ee). The ee was determined on an AS-H column (hexanes/isopropanol 99:1, flow $1.0 \mathrm{~mL} / \mathrm{min}$ ) with $\mathrm{t}_{\mathrm{r}}$ (major) $9.8 \mathrm{~min}, \mathrm{t}_{\mathrm{r}}$ (minor) $10.6 \mathrm{~min}$.

$[\alpha]_{D}^{22}=-43.5\left(\mathrm{c}=0.97, \mathrm{CHCl}_{3}\right)$;

mp: $77-78^{\circ} \mathrm{C}$;

${ }^{1} \mathrm{H}$ NMR $\left(\mathrm{CDCl}_{3}, 500 \mathrm{MHz}\right) \delta$ 7.32-7.19 (m, 11H), 7.18-7.12 (m, 2H), 6.91-6.89 (m, 2H), $4.95(\mathrm{~d}, J=14.3 \mathrm{~Hz}, 1 \mathrm{H}), 4.88(\mathrm{~d}, J=14.3 \mathrm{~Hz}, 1 \mathrm{H}), 2.57-2.46(\mathrm{~m}, 2 \mathrm{H}), 2.23-2.18(\mathrm{~m}, 1 \mathrm{H})$, $1.75-1.36(\mathrm{~m}, 6 \mathrm{H}), 0.82(\mathrm{t}, J=7.3 \mathrm{~Hz}, 3 \mathrm{H})$;

${ }^{13} \mathrm{C} \mathrm{NMR}\left(\mathrm{CDCl}_{3}, 125 \mathrm{MHz}\right) \delta 175.8,142.3,142.2,137.8,129.3,129.0,128.9,128.3$, $128.3,128.2,127.8,127.2,125.6,53.0,43.6,35.9,32.6,29.2,26.2,12.1$;

IR (film) 2961, 2930, 1653, 1595, 1495, 1454, 1402, 1242, 1200, $748 \mathrm{~cm}^{-1}$;

HRMS (ESI) calcd for $\mathrm{C}_{26} \mathrm{H}_{30} \mathrm{NO}\left(\mathrm{M}+\mathrm{H}^{+}\right)$372.2322, found 372.2315.

(-)-N-Benzyl- $N$-phenyl-2-(cyclohexylmethyl)butanamide (Table 1, entry 4$)$. The compound was prepared according to the General Procedure, except that the reaction was run at r.t. (not $0^{\circ} \mathrm{C}$ ), with $( \pm)-N$-benzyl- $N$-phenyl-2-bromobutanamide (332 $\mathrm{mg}$, $1.00 \mathrm{mmol}$ ) and the organozinc reagent ( 1.0 M in DMI; $1.3 \mathrm{~mL}, 1.3 \mathrm{mmol})$. After chromatography on silica gel (hexanes/ethyl acetate 12:1), the title compound was isolated as a colorless oil: run 1: $211 \mathrm{mg}$ (60\%; 92\% ee); run 2: $195 \mathrm{mg}(56 \%$; $92 \%$ ee). The ee was determined on an AS-H column (hexanes/iso-propanol 99:1, flow $1.0 \mathrm{~mL} / \mathrm{min}$ ) with $\mathrm{t}_{\mathrm{r}}$ (major) $4.9 \mathrm{~min}, \mathrm{t}_{\mathrm{r}}$ (minor) $5.3 \mathrm{~min}$.

$[\alpha]_{D}^{22}=-4.8\left(\mathrm{c}=1.04, \mathrm{CHCl}_{3}\right)$;

${ }^{1} \mathrm{H} \mathrm{NMR}\left(\mathrm{CDCl}_{3}, 500 \mathrm{MHz}\right) \delta$ 7.33-7.19 (m, 8H), 6.95-6.91 (m, 2H), $4.99(\mathrm{~d}, J=14.0 \mathrm{~Hz}$, $1 \mathrm{H}), 4.83(\mathrm{~d}, J=14.0 \mathrm{~Hz}, 1 \mathrm{H}), 2.30-2.25(\mathrm{~m}, 1 \mathrm{H}), 1.68-1.53(\mathrm{~m}, 5 \mathrm{H}), 1.45-1.34(\mathrm{~m}, 3 \mathrm{H})$, $1.26-1.04(\mathrm{~m}, 5 \mathrm{H}), 0.82(\mathrm{t}, J=7.3 \mathrm{~Hz}, 3 \mathrm{H}), 0.71-0.62(\mathrm{~m}, 2 \mathrm{H})$; 
${ }^{13} \mathrm{C} \mathrm{NMR}\left(\mathrm{CDCl}_{3}, 125 \mathrm{MHz}\right) \delta 176.1,142.2,137.9,129.2,128.9,128.2,127.8,127.2,52.9$, 41.0, 40.8, 35.3, 33.6, 33.3, 26.5, 26.2, 26.2, 26.1, 12.2;

IR (film) 2922, 2849, 1653, 1595, 1495, 1449, $1400 \mathrm{~cm}^{-1}$;

HRMS (ESI) calcd for $\mathrm{C}_{24} \mathrm{H}_{31} \mathrm{NONa}\left(\mathrm{M}+\mathrm{Na}^{+}\right)$372.2298, found 372.2304.

(-)-N-Benzyl- $N$-phenyl-2-butyloctanamide (Table 1, entry 5). The compound was prepared according to the General Procedure with $( \pm)-N$-benzyl- $N$-phenyl-2bromohexanamide (360 mg, $1.00 \mathrm{mmol})$ and $\mathrm{HexZnBr}(\sim 1.0 \mathrm{M}$ in DMI; $1.3 \mathrm{~mL}, 1.3$ $\mathrm{mmol}$ ). After chromatography on silica gel (hexanes/ethyl acetate 12:1), the title compound was isolated as a colorless oil: run 1: $314 \mathrm{mg}(86 \% ; 96 \%$ ee); run 2: $306 \mathrm{mg}$ ( $84 \%$; 96\% ee). The ee was determined on an AD-H column (hexanes/iso-propanol 99:1, flow $1.0 \mathrm{~mL} / \mathrm{min}$ ) with $\mathrm{t}_{\mathrm{r}}$ (major) $10.9 \mathrm{~min}, \mathrm{t}_{\mathrm{r}}$ (minor) $12.6 \mathrm{~min}$.

$[\alpha]^{23}=-9.0\left(\mathrm{c}=1.27, \mathrm{CHCl}_{3}\right)$;

${ }^{1} \mathrm{H}$ NMR $\left(\mathrm{CDCl}_{3}, 500 \mathrm{MHz}\right) \delta$ 7.33-7.19 (m, 8H), 6.93-6.91 (m, 2H), $4.93(\mathrm{~d}, J=14.0 \mathrm{~Hz}$, $1 \mathrm{H}), 4.88(\mathrm{~d}, J=14.0 \mathrm{~Hz}, 1 \mathrm{H}), 2.25-2.19(\mathrm{~m}, 1 \mathrm{H}), 1.66-1.59(\mathrm{~m}, 2 \mathrm{H}), 1.37-1.08(\mathrm{~m}, 14 \mathrm{H})$, $0.88-0.83(\mathrm{~m}, 6 \mathrm{H})$;

${ }^{13} \mathrm{C}$ NMR $\left(\mathrm{CDCl}_{3}, 125 \mathrm{MHz}\right) \delta 176.2,142.2,137.9,129.2,128.9,128.9,128.2,127.7$, $127.2,52.9,42.1,33.2,32.9,31.7,29.7,29.3,27.5,22.7,22.6,14.1,13.9$;

IR (film) 2955, 2927, 2857, 1654, 1595, 1494, 1455, $1400 \mathrm{~cm}^{-1}$;

HRMS (ESI) calcd for $\mathrm{C}_{25} \mathrm{H}_{35} \mathrm{NONa}\left(\mathrm{M}+\mathrm{Na}^{+}\right) 388.2616$, found 388.2614.

(-)- $N$-Benzyl- $N$-phenyl-2-(3-phenylpropyl)hexanamide (Table 1, entry 6). The compound was prepared according to the General Procedure with $( \pm)-\mathrm{N}$-benzyl- $\mathrm{N}$ phenyl-2-bromohexanamide $(360 \mathrm{mg}, 1.00 \mathrm{mmol})$ and the organozinc reagent $(\sim 1.0 \mathrm{M}$ in DMI; $1.3 \mathrm{~mL}, 1.3 \mathrm{mmol}$ ). After chromatography on silica gel (hexanes/ethyl acetate 10:1), the title compound was isolated as a colorless oil: run 1: $319 \mathrm{mg}(80 \%$; $96 \%$ ee); run 2: $311 \mathrm{mg}$ ( $78 \%$; $96 \%$ ee). The ee was determined on an AD-H column (hexanes/isopropanol 99:1, flow $1.0 \mathrm{~mL} / \mathrm{min}$ ) with $\mathrm{t}_{\mathrm{r}}$ (major) $19.8 \mathrm{~min}, \mathrm{t}_{\mathrm{r}}$ (minor) $24.0 \mathrm{~min}$.

$[\alpha]_{D}^{23}=-25.3\left(\mathrm{c}=1.23, \mathrm{CHCl}_{3}\right)$;

${ }^{1} \mathrm{H}$ NMR $\left(\mathrm{CDCl}_{3}, 500 \mathrm{MHz}\right) \delta$ 7.33-7.19 (m, 11H), 7.18-7.11 (m, 2H), 6.91-6.88 (m, 2H), $4.93(\mathrm{~d}, J=14.0 \mathrm{~Hz}, 1 \mathrm{H}), 4.89(\mathrm{~d}, J=14.0 \mathrm{~Hz}, 1 \mathrm{H}), 2.56-2.46(\mathrm{~m}, 2 \mathrm{H}), 2.29-2.23(\mathrm{~m}, 1 \mathrm{H})$, $1.74-1.32(\mathrm{~m}, 6 \mathrm{H}), 1.22-1.09(\mathrm{~m}, 4 \mathrm{H}), 0.85(\mathrm{t}, J=7.0 \mathrm{~Hz}, 3 \mathrm{H})$;

${ }^{13} \mathrm{C} \mathrm{NMR}\left(\mathrm{CDCl}_{3}, 125 \mathrm{MHz}\right) \delta 175.9,142.3,142.2,137.8,129.3,128.9,128.3,128.3$, $128.2,127.8,127.2,125.6,52.9,42.0,36.0,32.8,32.8,29.7,29.2,22.7,13.9$;

IR (film) 2952, 2929, 2857, 1654, 1595, 1495, 1454, 1401, 1247, 1196, $748 \mathrm{~cm}^{-1}$;

HRMS (ESI) calcd for $\mathrm{C}_{28} \mathrm{H}_{34} \mathrm{NO}\left(\mathrm{M}+\mathrm{H}^{+}\right) 400.2635$, found 400.2622.

(-)-N-Benzyl- $N$-phenyl-2,4-dimethylpentanamide (Table 1, entry 7). The compound was prepared according to the General Procedure with ( \pm )- $N$-benzyl- $N$ phenyl-2-bromo-4-methylpentanamide (360 mg, $1.00 \mathrm{mmol}$ ) and MeZnI ( 1.0 M in DMI; $1.3 \mathrm{~mL}, 1.3 \mathrm{mmol}$ ). After chromatography on silica gel (hexanes/ethyl acetate 12:1), the title compound was isolated as a colorless oil: run 1: $233 \mathrm{mg}(79 \% ; 87 \%$ ee); run 2: 226 $\mathrm{mg}(77 \% ; 87 \%$ ee). The ee was determined on an AD-H column (hexanes/iso-propanol 99:1, flow $1.0 \mathrm{~mL} / \mathrm{min}$ ) with $\mathrm{t}_{\mathrm{r}}$ (major) $12.4 \mathrm{~min}, \mathrm{t}_{\mathrm{r}}($ minor) $13.5 \mathrm{~min}$.

$$
[\alpha]^{23}=-43.3\left(\mathrm{c}=1.08, \mathrm{CHCl}_{3}\right) \text {; }
$$


${ }^{1} \mathrm{H}$ NMR $\left(\mathrm{CDCl}_{3}, 500 \mathrm{MHz}\right) \delta$ 7.34-7.18 (m, 8H), 6.98-6.96 (m, 2H), $4.91(\mathrm{~d}, J=14.0 \mathrm{~Hz}$, $1 \mathrm{H}), 4.86(\mathrm{~d}, J=14.3 \mathrm{~Hz}, 1 \mathrm{H}), 2.44-2.37(\mathrm{~m}, 1 \mathrm{H}), 1.68-1.62(\mathrm{~m}, 1 \mathrm{H}), 1.49(\mathrm{ddd}, J=6.4,6.7$, $6.7 \mathrm{~Hz}, 1 \mathrm{H}), 1.18-1.09(\mathrm{~m}, 1 \mathrm{H}), 1.04(\mathrm{~d}, J=6.7 \mathrm{~Hz}, 3 \mathrm{H}), 0.73(\mathrm{~d}, J=6.4 \mathrm{~Hz}, 3 \mathrm{H}), 0.69(\mathrm{~d}, J=$ $6.7 \mathrm{~Hz}, 3 \mathrm{H})$;

${ }^{13} \mathrm{C}$ NMR $\left(\mathrm{CDCl}_{3}, 125 \mathrm{MHz}\right) \delta 176.8,142.3,137.8,129.3,128.8,128.5,128.3,127.8$, 127.2, 52.8, 43.6, 34.6, 25.7, 22.7, 22.5, 18.2;

IR (film) 2957, 2932, 1656, 1595, 1494, 1455, 1399, 1254, $1200 \mathrm{~cm}^{-1}$;

HRMS (ESI) calcd for $\mathrm{C}_{20} \mathrm{H}_{26} \mathrm{NO}\left(\mathrm{M}+\mathrm{H}^{+}\right)$296.2009, found 296.2003.

(-)- $N$-Benzyl- $N$-phenyl-2-ethyl-6-methylhept-5-enamide (Table 1, entry 8). The compound was prepared according to the General Procedure with ( \pm )- $N$-benzyl- $N$ phenyl-2-bromobutanamide $(332 \mathrm{mg}, 1.00 \mathrm{mmol})$ and the organozinc reagent $(\sim 1.0 \mathrm{M}$ in DMI; $1.3 \mathrm{~mL}, 1.3 \mathrm{mmol}$ ). After chromatography on silica gel (hexanes/ethyl acetate 15:1), the title compound was isolated as a colorless oil: run 1: $271 \mathrm{mg}(81 \%$; $95 \%$ ee); run 2: $248 \mathrm{mg}$ (74\%; 95\% ee). The ee was determined on an AS-H column (hexanes/isopropanol 99:1, flow $1.0 \mathrm{~mL} / \mathrm{min}$ ) with $\mathrm{t}_{\mathrm{r}}$ (major) $5.6 \mathrm{~min}, \mathrm{t}_{\mathrm{r}}$ (minor) $6.2 \mathrm{~min}$.

$[\alpha]^{22}=-42.4\left(\mathrm{c}=1.26, \mathrm{CHCl}_{3}\right)$;

${ }^{1} \mathrm{H}$ NMR $\left(\mathrm{CDCl}_{3}, 500 \mathrm{MHz}\right)$ d 7.34-7.20 (m, 8H), 6.95-6.92 (m, 2H), $4.95(\mathrm{~d}, J=14.0 \mathrm{~Hz}$, $1 \mathrm{H}), 4.92-4.90(\mathrm{~m}, 1 \mathrm{H}), 4.87(\mathrm{~d}, J=14.0 \mathrm{~Hz}, 1 \mathrm{H}), 2.22-2.17(\mathrm{~m}, 1 \mathrm{H}), 1.96-1.81(\mathrm{~m}, 2 \mathrm{H})$, $1.70-1.63(\mathrm{~m}, 2 \mathrm{H}), 1.61(\mathrm{~d}, J=0.9 \mathrm{~Hz}, 3 \mathrm{H}), 1.53(\mathrm{~s}, 3 \mathrm{H}), 1.43-1.35(\mathrm{~m}, 2 \mathrm{H}), 0.82(\mathrm{t}, J=7.3$ $\mathrm{Hz}, 3 \mathrm{H})$;

${ }^{13} \mathrm{C}$ NMR $\left(\mathrm{CDCl}_{3}, 125 \mathrm{MHz}\right) \delta 175.8,142.2,137.9,131.6,129.2,129.0,128.9,128.2$, $127.7,127.2,124.0,52.9,43.2,33.0,25.9,25.8,25.6,17.6,12.1$;

IR (film) 2963, 2926, 1654, 1595, 1495, 1455, 1402, 1247, $1200 \mathrm{~cm}^{-1}$;

HRMS (ESI) calcd for $\mathrm{C}_{23} \mathrm{H}_{30} \mathrm{NO}\left(\mathrm{M}+\mathrm{H}^{+}\right)$336.2322, found 336.2325.

(-)-N-Benzyl-N-phenyl-6-(benzyloxy)-2-ethylhexanamide (Table 1, entry 9). The compound was prepared according to the General Procedure with $( \pm)-\mathrm{N}$-benzyl- $\mathrm{N}$ phenyl-2-bromobutanamide $(332 \mathrm{mg}, 1.00 \mathrm{mmol})$ and the organozinc reagent $(\sim 1.0 \mathrm{M}$ in DMI; $1.3 \mathrm{~mL}, 1.3 \mathrm{mmol}$ ). After chromatography on silica gel (hexanes/ethyl acetate 5:1), the title compound was isolated as a colorless oil: run 1: $317 \mathrm{mg}(76 \% ; 96 \%$ ee); run 2: $324 \mathrm{mg}(78 \%$; $96 \%$ ee). The ee was determined on an AD-H column (hexanes/isopropanol 97:3, flow $1.0 \mathrm{~mL} / \mathrm{min}$ ) with $\mathrm{t}_{\mathrm{r}}$ (major) $18.9 \mathrm{~min}, \mathrm{t}_{\mathrm{r}}$ (minor) $25.6 \mathrm{~min}$.

$[\alpha]^{23}=-30.9\left(\mathrm{c}=1.35, \mathrm{CHCl}_{3}\right)$;

${ }^{1} \mathrm{H}$ NMR $\left(\mathrm{CDCl}_{3}, 500 \mathrm{MHz}\right) \delta$ 7.36-7.20 (m, 13H), 6.93-6.91 (m, 2H), $4.96(\mathrm{~d}, J=14.0$ $\mathrm{Hz}, 1 \mathrm{H}), 4.85(\mathrm{~d}, J=14.0 \mathrm{~Hz}, 1 \mathrm{H}), 4.48(\mathrm{~s}, 2 \mathrm{H}), 3.43-3.38(\mathrm{~m}, 2 \mathrm{H}), 2.21-2.16(\mathrm{~m}, 1 \mathrm{H}), 1.70-$ $1.62(\mathrm{~m}, 2 \mathrm{H}), 1.54-1.23(\mathrm{~m}, 6 \mathrm{H}), 0.81(\mathrm{t}, J=7.3 \mathrm{~Hz}, 3 \mathrm{H})$;

${ }^{13} \mathrm{C}$ NMR $\left(\mathrm{CDCl}_{3}, 125 \mathrm{MHz}\right) \delta 175.9,142.2,138.6,137.8,129.3,129.0,128.9,128.3$, 128.3, 127.8, 127.6, 127.4, 127.2, 72.8, 70.1, 52.9, 43.6, 32.8, 29.8, 26.2, 24.1, 12.1; $\mathrm{cm}^{-1}$;

IR (film) 2960, 2932, 2859, 1650, 1595, 1495, 1455, 1401, 1242, 1202, 1101, 1080, 736, 408

HRMS (ESI) calcd for $\mathrm{C}_{28} \mathrm{H}_{33} \mathrm{NO}_{2} \mathrm{Na}\left(\mathrm{M}+\mathrm{Na}^{+}\right)$438.2404, found 438.2419.

(-)- $N$-Benzyl- $N$-phenyl-5-(1,3-dioxolan-2-yl)-2-methylpentanamide (Table 1, entry 10). The compound was prepared according to the General Procedure with $( \pm)-N$ benzyl- $N$-phenyl-2-bromopropanamide $(318 \mathrm{mg}, 1.00 \mathrm{mmol})$ and the organozinc 
reagent ( 1.0 M in DMI; $1.3 \mathrm{~mL}, 1.3 \mathrm{mmol})$. After chromatography on silica gel (hexanes/ethyl acetate 3:1), the title compound was isolated as a colorless solid: run 1: $234 \mathrm{mg}$ (66\%; 77\% ee); run 2: $211 \mathrm{mg}(60 \%$; $>98 \%$ ee; obtained after recrystallization from pentanes/diethyl ether). The ee was determined on an AD-H column (hexanes/iso-propanol 90:10, flow $0.9 \mathrm{~mL} / \mathrm{min}$ ) with $\mathrm{t}_{\mathrm{r}}$ (major) $11.3 \mathrm{~min}, \mathrm{t}_{\mathrm{r}}$ (minor) 15.2 $\min$.

$[\alpha]^{22}=-0.6\left(\mathrm{c}=0.98, \mathrm{CHCl}_{3}\right)$;

mp: $92-93^{\circ} \mathrm{C}$ (recrystallized from pentanes/diethyl ether; $>98 \%$ ee);

${ }^{1} \mathrm{H} \mathrm{NMR}\left(\mathrm{CDCl}_{3}, 500 \mathrm{MHz}\right)$ d 7.34-7.17 (m, 8H), 6.96-6.93 (m, 2H), $4.91(\mathrm{~d}, J=14.3 \mathrm{~Hz}$, $1 \mathrm{H}), 4.84(\mathrm{~d}, J=14.0 \mathrm{~Hz}, 1 \mathrm{H}), 4.40-4.38(\mathrm{~m}, 1 \mathrm{H}), 4.07-4.04(\mathrm{~m}, 2 \mathrm{H}), 3.72-3.66(\mathrm{~m}, 2 \mathrm{H})$, 2.36-2.31 (m, 1H), 2.09-1.99 (m, 1H), 1.82-1.76 (m, 1H), 1.59-1.53 (m, 1H), 1.49-1.37 (m, $2 \mathrm{H}), 1.33-1.29(\mathrm{~m}, 1 \mathrm{H}), 1.04(\mathrm{~d}, J=6.7 \mathrm{~Hz}, 3 \mathrm{H})$;

${ }^{13} \mathrm{C} \mathrm{NMR}\left(\mathrm{CDCl}_{3}, 125 \mathrm{MHz}\right) \delta 176.3,142.2,137.7,129.4,128.8,128.5,128.3,127.8$, 127.2, 102.1, 66.8, 66.8, 52.9, 36.4, 33.0, 28.8, 25.8, 18.3;

IR (film) 2961, 2852, 1654, 1594, 1495, 1457, 1400, 1258, 1143, 999, $406 \mathrm{~cm}^{-1}$;

HRMS (ESI) calcd for $\mathrm{C}_{22} \mathrm{H}_{28} \mathrm{NO}_{3}\left(\mathrm{M}+\mathrm{H}^{+}\right)$354.2064, found 354.2054.

(-)-N-Benzyl-N-phenyl-2-ethyl-6-(1,3-dioxoisoindolin-2-yl)hexanamide (Table 1, entry 11). The compound was prepared according to the General Procedure with $( \pm)-\mathrm{N}-$ benzyl- $N$-phenyl-2-bromobutanamide $(332 \mathrm{mg}, 1.00 \mathrm{mmol})$ and the organozinc reagent ( 1.0 M in DMI; $1.3 \mathrm{~mL}, 1.3 \mathrm{mmol})$. After chromatography on silica gel (hexanes/ethyl acetate 5:2), the title compound was isolated as a colorless solid: run 1: $234 \mathrm{mg}$ (51\%; $96 \%$ ee); run 2: $231 \mathrm{mg}$ (51\%; 96\% ee). The ee was determined on an AD-H column (hexanes/iso-propanol 80:20, flow $0.9 \mathrm{~mL} / \mathrm{min}$ ) with $\mathrm{t}_{\mathrm{r}}$ (major) $16.1 \mathrm{~min}, \mathrm{t}_{\mathrm{r}}$ (minor) 21.9 $\min$.

$[\alpha]_{\mathrm{D}}^{23}=-41.3\left(\mathrm{c}=1.18, \mathrm{CHCl}_{3}\right)$;

mp: $68-69^{\circ} \mathrm{C}$;

${ }^{1} \mathrm{H}$ NMR $\left(\mathrm{CDCl}_{3}, 500 \mathrm{MHz}\right) \delta$ 7.86-7.83 (m, 2H), 7.74-7.70 (m, 2H), 7.29-7.18 (m, 8H), 6.93-6.92 (m, 2H), $5.03(\mathrm{~d}, J=14.0 \mathrm{~Hz}, 1 \mathrm{H}), 4.76(\mathrm{~d}, J=14.0 \mathrm{~Hz}, 1 \mathrm{H}), 3.64-3.61(\mathrm{~m}, 2 \mathrm{H})$, 2.18-2.12 (m, 1H), 1.69-1.50 (m, 4H), 1.39-1.16 (m, 4H), $0.79(\mathrm{t}, J=7.2 \mathrm{~Hz}, 3 \mathrm{H})$;

${ }^{13} \mathrm{C} \mathrm{NMR}\left(\mathrm{CDCl}_{3}, 125 \mathrm{MHz}\right) \delta 175.7,168.4,142.1,137.7,133.9,132.1,129.3,129.1$, $128.9,128.3,127.8,127.2,123.1,53.0,43.6,37.9,32.7,28.8,26.3,25.1,12.1$;

IR (film) 2961, 2934, 1772, 1713, 1652, 1595, 1495, 1437, 1397, 1363, 721, $701 \mathrm{~cm}^{-1}$;

HRMS (ESI) calcd for $\mathrm{C}_{29} \mathrm{H}_{30} \mathrm{~N}_{2} \mathrm{O}_{3} \mathrm{Na}\left(\mathrm{M}+\mathrm{Na}^{+}\right)$477.2149, found 477.2159.

(-)- $N$-Benzyl- $N$-phenyl-7-cyano-2-ethylheptanamide (Table 1, entry 12). The compound was prepared according to the General Procedure with ( \pm )- $\mathrm{N}$-benzyl- $\mathrm{N}$ phenyl-2-bromobutanamide $(332 \mathrm{mg}, 1.00 \mathrm{mmol})$ and the organozinc reagent $(\sim 1.0 \mathrm{M}$ in DMI; $1.3 \mathrm{~mL}, 1.3 \mathrm{mmol}$ ). After chromatography on silica gel (hexanes/ethyl acetate 5:1 $\rightarrow 3: 1)$, the title compound was isolated as a colorless solid: run 1: $255 \mathrm{mg}$ (73\%; 93\% ee); run 2: $234 \mathrm{mg}(67 \% ; 92 \%$ ee). The ee was determined on an AS-H column (hexanes/isopropanol 97:3, flow $1.0 \mathrm{~mL} / \mathrm{min}$ ) with $\mathrm{t}_{\mathrm{r}}$ (major) $22.0 \mathrm{~min}, \mathrm{t}_{\mathrm{r}}$ (minor) $25.4 \mathrm{~min}$.

$[\alpha]_{D}^{23}=-30.8\left(\mathrm{c}=1.44, \mathrm{CHCl}_{3}\right)$;

mp: $83-86^{\circ} \mathrm{C}$; 
${ }^{1} \mathrm{H}$ NMR $\left(\mathrm{CDCl}_{3}, 500 \mathrm{MHz}\right) \delta$ 7.35-7.19 (m, 8H), 6.93-6.91 (m, 2H), $4.95(\mathrm{~d}, J=14.0 \mathrm{~Hz}$, $1 \mathrm{H}), 4.86(\mathrm{~d}, J=14.0 \mathrm{~Hz}, 1 \mathrm{H}), 2.28(\mathrm{t}, J=7.3 \mathrm{~Hz}, 2 \mathrm{H}), 2.21-2.15(\mathrm{~m}, 1 \mathrm{H}), 1.68-1.57(\mathrm{~m}, 4 \mathrm{H})$, $1.41-1.16(\mathrm{~m}, 6 \mathrm{H}), 0.81(\mathrm{t}, J=7.3 \mathrm{~Hz}, 3 \mathrm{H})$;

${ }^{13} \mathrm{C} \mathrm{NMR}\left(\mathrm{CDCl}_{3}, 125 \mathrm{MHz}\right) \delta 175.6,142.2,137.7,129.4,128.9,128.3,127.9,127.3$, $119.7,53.0,43.4,32.4,28.6,26.5,26.1,25.1,17.0,12.1$;

IR (film) 2960, 2933, 1652, 1595, 1495, 1456, 1403, 1242, $1200 \mathrm{~cm}^{-1}$;

HRMS (ESI) calcd for $\mathrm{C}_{23} \mathrm{H}_{29} \mathrm{~N}_{2} \mathrm{O}\left(\mathrm{M}+\mathrm{H}^{+}\right)$349.2274, found 349.2274.

Competition experiment (eq 5 ; $\boldsymbol{n}$-octyl bromide). In a glove box, a 4 -mL vial was charged with $\mathrm{NiCl}_{2} \bullet$ glyme ( $\left.5.5 \mathrm{mg}, 25 \mu \mathrm{mol}\right),(R)-(i-\mathrm{Pr})-\mathrm{Pybox}(9.8 \mathrm{mg}, 33 \mu \mathrm{mol}),( \pm)-\mathrm{N}-$ benzyl- $N$-phenyl-2-bromobutanamide $(83 \mathrm{mg}, 0.25 \mathrm{mmol}), n$-octyl bromide $(43 \mu \mathrm{L}, 0.25$ $\mathrm{mmol})$, DMI $(0.55 \mathrm{~mL})$, and THF $(0.12 \mathrm{~mL})$. The orange solution was stirred for 20 minutes, and then the vial was closed with a septum cap, removed from the glove box, fitted with an argon inlet, and placed in a $0^{\circ} \mathrm{C}$ bath. The mixture was stirred for 10 minutes, and then HexZnBr ( 1.0 M in DMI; $0.33 \mathrm{~mL}, 0.33 \mathrm{mmol})$ was added. The resulting dark-brown reaction mixture was stirred for $13 \mathrm{~h}$ at $0{ }^{\circ} \mathrm{C}$. Then, the excess organozinc reagent was quenched by the addition of ethanol $(0.5 \mathrm{~mL})$, and $n$-decane (calibrated internal standard; $48.8 \mu \mathrm{L}, 0.25 \mathrm{mmol}$ ) was added. The brown mixture was passed through a plug of silica gel (eluted with $\mathrm{Et}_{2} \mathrm{O}$ ) to remove inorganic salts and most of the DMI. The filtrate was analyzed by GC to determine the conversion.

The enantiomeric excess was determined on a Daicel Chiralpak ${ }^{\circledR}$ AD-H column in hexanes/iso-propanol (99:1, flow $1.0 \mathrm{~mL} / \mathrm{min}$ ) after filtration through silica gel with $10 \%$ iso-propanol in hexanes. Run 1: 87\% (-)-N-benzyl- $N$-phenyl-2-ethyloctanamide (HPLC: hexanes/iso-propanol 99:1, flow $1.0 \mathrm{~mL} / \mathrm{min}, 95 \%$ ee), $92 \%$ n-octylbromide, and no $n$ tetradecane detectable; Run 2: 86\% (-)-N-benzyl- $\mathrm{N}$-phenyl-2-ethyloctanamide (HPLC: hexanes/iso-propanol 99:1, flow $1.0 \mathrm{~mL} / \mathrm{min}, 96 \%$ ee), $97 \%$-octylbromide, and no $n$ tetradecane detectable; Run 3: 90\% (-)- $N$-benzyl- $N$-phenyl-2-ethyloctanamide (HPLC: hexanes/iso-propanol 99:1, flow $1.0 \mathrm{~mL} / \mathrm{min}, 96 \%$ ee), $97 \%$ n-octylbromide, and no $n$ tetradecane detectable;

Competition experiment (eq 5; cyclooctyl bromide). In a glove box, a 4-mL vial was charged with $\mathrm{NiCl}_{2} \cdot$ glyme $(5.5 \mathrm{mg}, 25 \mu \mathrm{mol}),(R)-(i-\mathrm{Pr})-\mathrm{Pybox}(9.8 \mathrm{mg}, 33 \mu \mathrm{mol}),( \pm)-\mathrm{N}-$ benzyl- $N$-phenyl-2-bromobutanamide $(83 \mathrm{mg}, 0.25 \mathrm{mmol})$, cyclooctyl bromide $(37 \mu \mathrm{L}$, $0.25 \mathrm{mmol})$, DMI $(0.55 \mathrm{~mL})$, and THF $(0.12 \mathrm{~mL})$. The resulting orange solution was stirred for 20 minutes, and then the vial was closed with a septum cap, removed from the glove box, fitted with an argon inlet, and placed in a $0{ }^{\circ} \mathrm{C}$ bath. The mixture was stirred for 10 minutes, and then HexZnBr ( 1.0 M in DMI; $0.33 \mathrm{~mL}, 0.33 \mathrm{mmol})$ was added. The resulting dark-brown reaction mixture was stirred for $13 \mathrm{~h}$ at $0{ }^{\circ} \mathrm{C}$. Then, the excess organozinc reagent was quenched by the addition of ethanol $(0.5 \mathrm{~mL})$, and $n$ decane (calibrated internal standard; $48.8 \mu \mathrm{L}, 0.25 \mathrm{mmol}$ ) was added. The brown mixture was passed through a plug of silica gel (eluted with $\mathrm{Et}_{2} \mathrm{O}$ ) to remove inorganic salts and most of the DMI. The filtrate was analyzed by GC to determine the conversion. The enantiomeric excess was determined on a Daicel Chiralpak ${ }^{\oplus} \mathrm{AD}-\mathrm{H}$ column in hexanes/iso-propanol (99:1, flow $1.0 \mathrm{~mL} / \mathrm{min}$ ) after filtration through silica gel with 10\% iso-propanol in hexanes. Run 1: 89\% (-)- $N$-benzyl- $N$-phenyl-2ethyloctanamide (HPLC: hexanes/iso-propanol 99:1, flow $1.0 \mathrm{~mL} / \mathrm{min}, 96 \%$ ee), $>98 \%$ cyclooctyl bromide, and no hexylcyclooctane detectable; Run 2: 84\% (-)-N-benzyl-N- 
phenyl-2-ethyloctanamide (HPLC: hexanes/iso-propanol 99:1, flow $1.0 \mathrm{~mL} / \mathrm{min}, 96 \%$ ee), >98\% cyclooctyl bromide, and no hexylcyclooctane detectable; Run 3: 84\% (-)-Nbenzyl- $N$-phenyl-2-ethyloctanamide (HPLC: hexanes/iso-propanol 99:1, flow 1.0 $\mathrm{mL} / \mathrm{min}, 96 \%$ ee), $>98 \%$ cyclooctyl bromide, and no hexylcyclooctane detectable.

Determination if the $\alpha$-bromo amide is being kinetically resolved (eq 6). In a glove box, a 4-mL vial was charged with $\mathrm{NiCl}_{2} \cdot$ glyme ( $\left.5.5 \mathrm{mg}, 25 \mu \mathrm{mol}\right),(R)-(i-\mathrm{Pr})-$ Pybox $(9.8 \mathrm{mg}, 33 \mu \mathrm{mol})$, and ( \pm )-N-benzyl- $N$-phenyl-2-bromobutanamide $(83 \mathrm{mg}, 0.25$ $\mathrm{mmol})$, DMI $(0.55 \mathrm{~mL})$, and THF $(0.12 \mathrm{~mL})$. The resulting orange solution was stirred for 20 minutes, and then the vial was closed with a septum cap, removed from the glove box, fitted with an argon inlet, and placed in a $0{ }^{\circ} \mathrm{C}$ bath. The mixture was stirred for 10 minutes, and then $\mathrm{HexZnBr}(\sim 1.0 \mathrm{M}$ in DMI; $0.20 \mathrm{~mL}, 0.20 \mathrm{mmol})$ was added. The resulting dark-brown reaction mixture was stirred for $13 \mathrm{~h}$ at $0{ }^{\circ} \mathrm{C}$. Then, the excess organozinc reagent was quenched by the addition of ethanol $(0.5 \mathrm{~mL})$, and $n$-decane (calibrated internal standard; $48.8 \mu \mathrm{L}, 0.25 \mathrm{mmol}$ ) was added. The brown mixture was passed through a plug of silica gel (eluted with $\mathrm{Et}_{2} \mathrm{O}$ ) to remove inorganic salts and most of the DMI. The filtrate was analyzed by GC to determine the conversion. The enantiomeric excess was determined on a Daicel Chiralpak ${ }^{\oplus} \mathrm{AD}-\mathrm{H}$ column in hexanes/iso-propanol (99:1, flow $1.0 \mathrm{~mL} / \mathrm{min}$ ) after filtration through silica gel with $10 \%$ iso-propanol in hexanes. Run 1: 53\% (-)-N-benzyl- $N$-phenyl-2-ethyloctanamide (HPLC: hexanes/iso-propanol 99:1, flow $1.0 \mathrm{~mL} / \mathrm{min}, 96 \%$ ee) and $46 \%$ ( \pm )- $N$-benzyl- $N$-phenyl2-bromobutanamide (HPLC: hexanes/iso-propanol 99:1, flow $1.0 \mathrm{~mL} / \mathrm{min}$, racemic); Run 2: 47\% (-)-N-benzyl- $\mathrm{N}$-phenyl-2-ethyloctanamide (HPLC: hexanes/iso-propanol 99:1, flow $1.0 \mathrm{~mL} / \mathrm{min}, 96 \%$ ee), $46 \%$ ( \pm )- $N$-benzyl- $N$-phenyl-2-bromobutanamide (HPLC: hexanes/iso-propanol 99:1, flow $1.0 \mathrm{~mL} / \mathrm{min}$, racemic).

(R)-(-)-2-Ethyloctan-1-ol (eq 7). ${ }^{3}$ A 25-mL flask was charged with (+)-N-benzyl- $N$ phenyl-2-ethyloctanamide $(169 \mathrm{mg}, 0.500 \mathrm{mmol})$, evacuated, and back-filled with argon. THF (5 mL) and $\mathrm{LiAlH}_{4}\left(1.0 \mathrm{M}\right.$ in $\mathrm{Et}_{2} \mathrm{O}$ (Aldrich); $\left.1.0 \mathrm{~mL}, 1.0 \mathrm{mmol}\right)$ were then added. The resulting colorless solution was stirred for 12 hours, and then the reaction was quenched by the slow addition of ethanol (until the gas evolution ceased). The gel-like reaction mixture was passed through a short plug of silica gel (eluted with $150 \mathrm{~mL}$ of $\mathrm{Et}_{2} \mathrm{O}$ ). The filtrate was concentrated, and the residue was purified by chromatography on silica gel (hexanes/ethyl acetate 10:1), which furnished $72 \mathrm{mg}(91 \%)$ of the title compound as a colorless oil. The ee was determined by chiral GC to be $96 \%$; $\mathrm{CP}-$ Chirasil-Dex CB, $\mathrm{t}_{\mathrm{r}}$ (minor) $52.3 \mathrm{~min}, \mathrm{t}_{\mathrm{r}}$ (major) $53.3 \mathrm{~min}$; heating program: $60^{\circ} \mathrm{C} \rightarrow 130$ ${ }^{\circ} \mathrm{C} @ 1{ }^{\circ} \mathrm{C} / \mathrm{min}$. In the second run, the product was obtained in $87 \%$ yield $(69 \mathrm{mg})$ and $96 \%$ ee.

$[\alpha]^{23}=-3.0\left(\mathrm{c}=1.14, \mathrm{CHCl}_{3}\right)$;

${ }^{1} \mathrm{H} \mathrm{NMR}\left(\mathrm{CDCl}_{3}, 500 \mathrm{MHz}\right) \delta$ 3.55-3.54 (m, 2H), 1.42-1.19 (m, 14H), $0.90(\mathrm{t}, J=7.3 \mathrm{~Hz}$, $3 \mathrm{H}), 0.88(\mathrm{t}, J=7.0 \mathrm{~Hz}, 3 \mathrm{H})$;

${ }^{13} \mathrm{C}$ NMR $\left(\mathrm{CDCl}_{3}, 125 \mathrm{MHz}\right) \delta$ 65.3, 42.0, 31.9, 30.4, 29.7, 26.8, 23.3, 22.7, 14.1, 11.1; IR (film) 3327, 2959, 2874, 2857, 1462, $1038 \mathrm{~cm}^{-1}$. 


\section{References}

(1) Pangborn, A. B.; Giardello, M. A.; Grubbs, R. H.; Rosen, R. K.; Timmers, F. J. Organometallics 1996, 15, 1518-1520.

(2) This is based on the procedure of Huo: Huo, S. Org. Lett. 2003, 5, 423-425.

(3) (a) Weitzel, G.; Jackisch, R.; Meier-Gerwert, H.; Zinser, D. Hoppe-Seyler's Z. Physiol. Chem. 1972, 641-653. (b) Garcia-Ruiz, V.; Woodward, S. Tetrahedron: Asymmetry 2002, 13, 2177-2180. (c) Norsikian, S.; Baudry, M.; Normant, J. F. Tetrahedron Lett. 2000, 41, 6575-6578. 


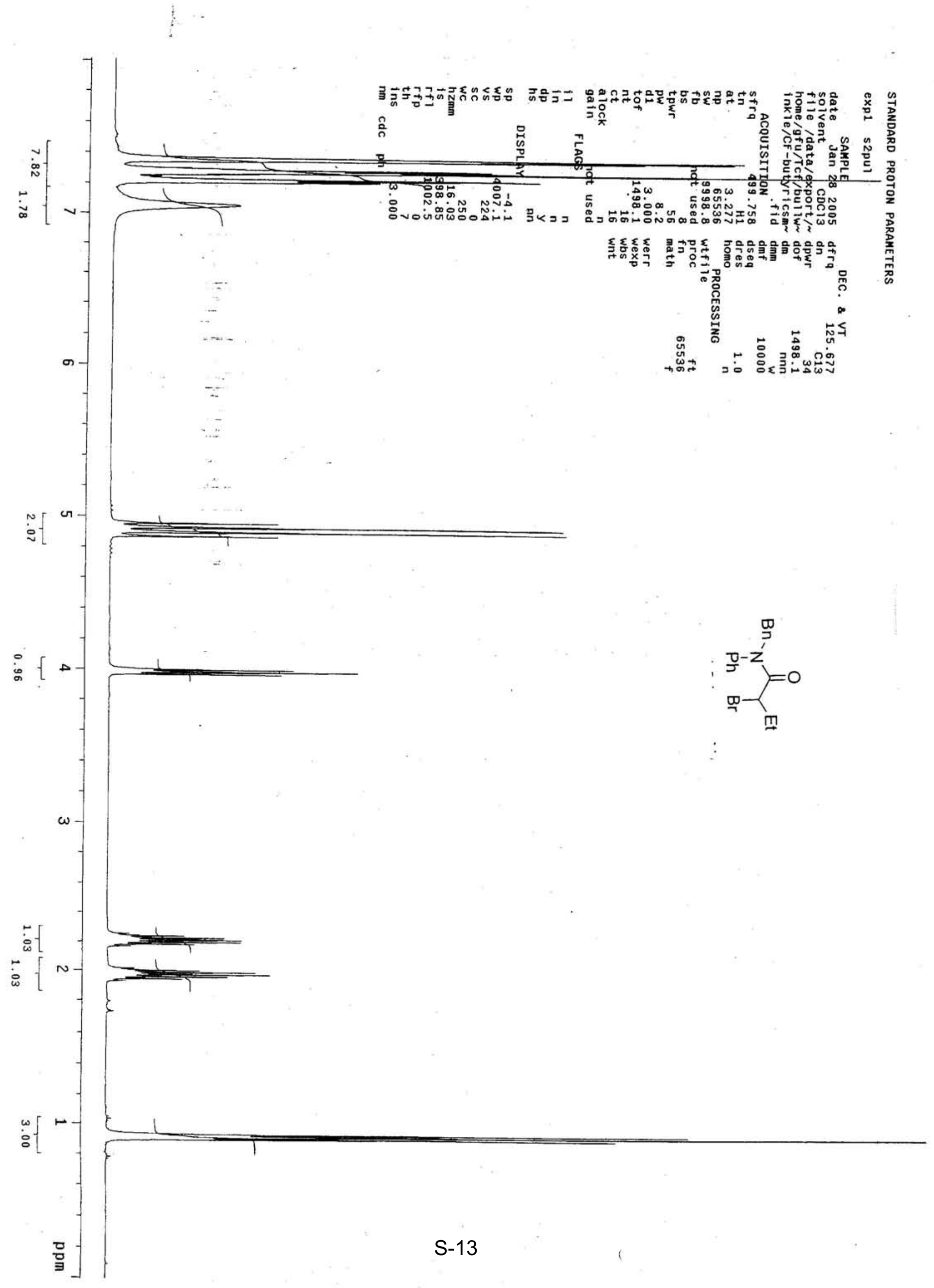




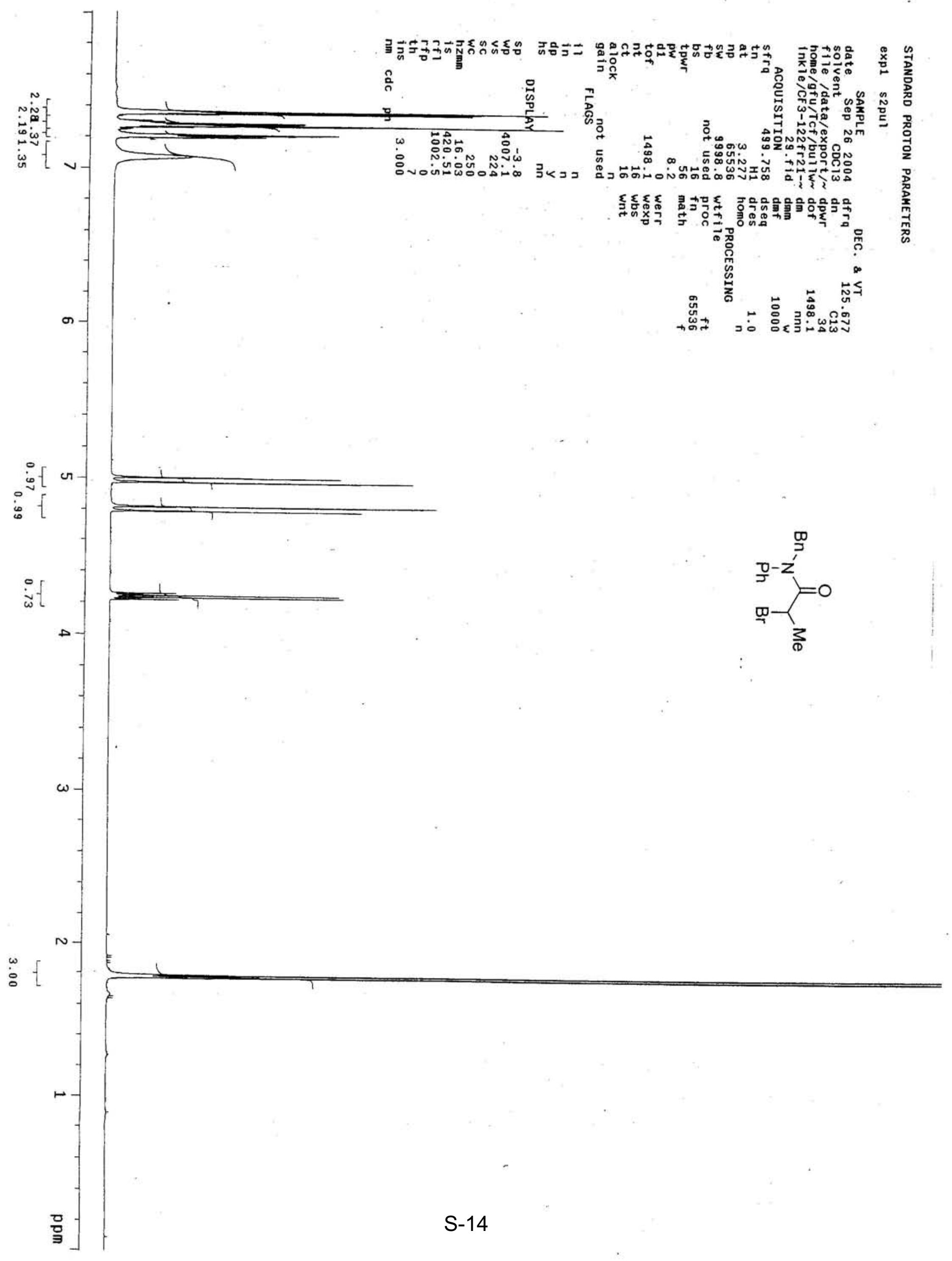




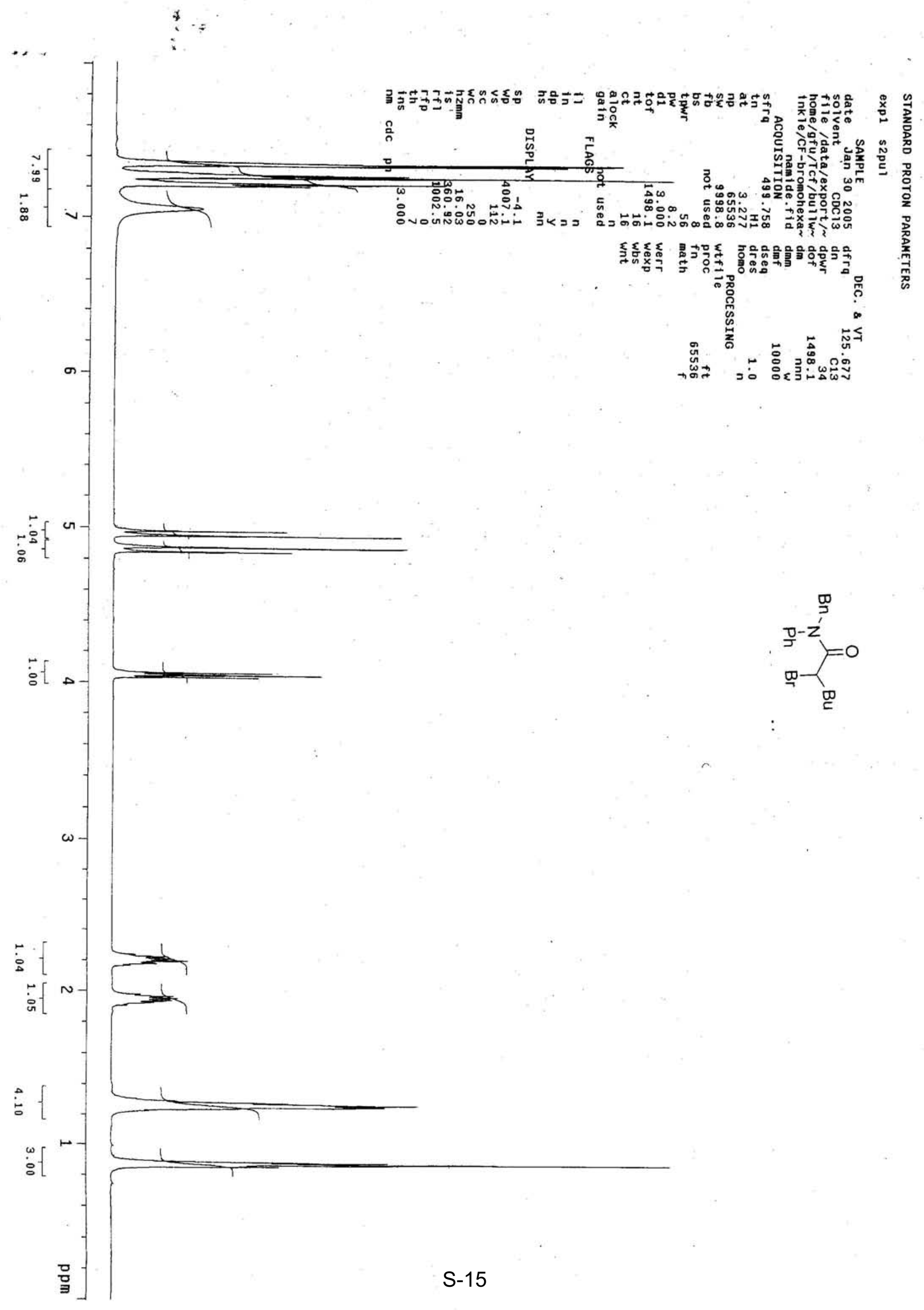




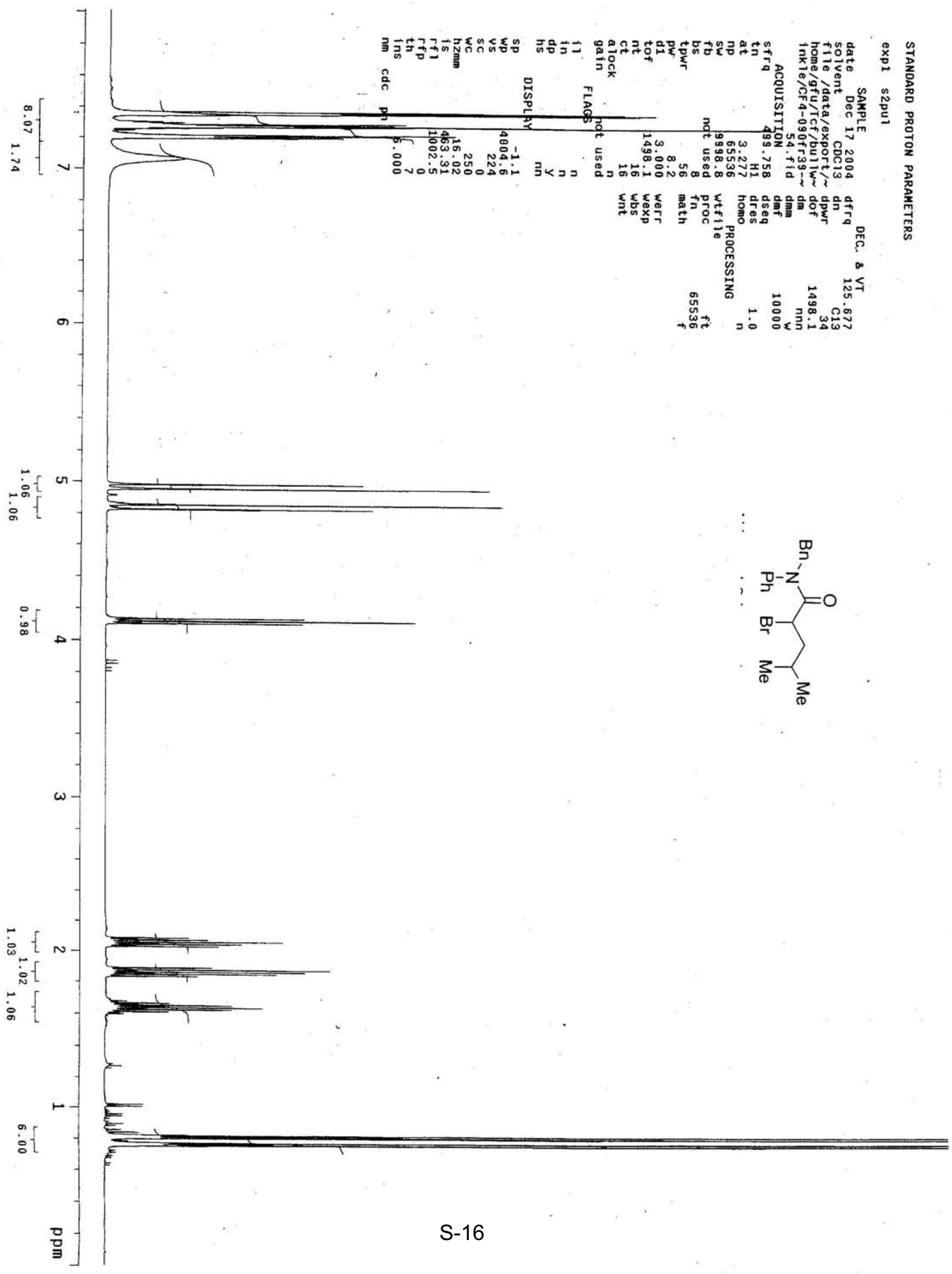




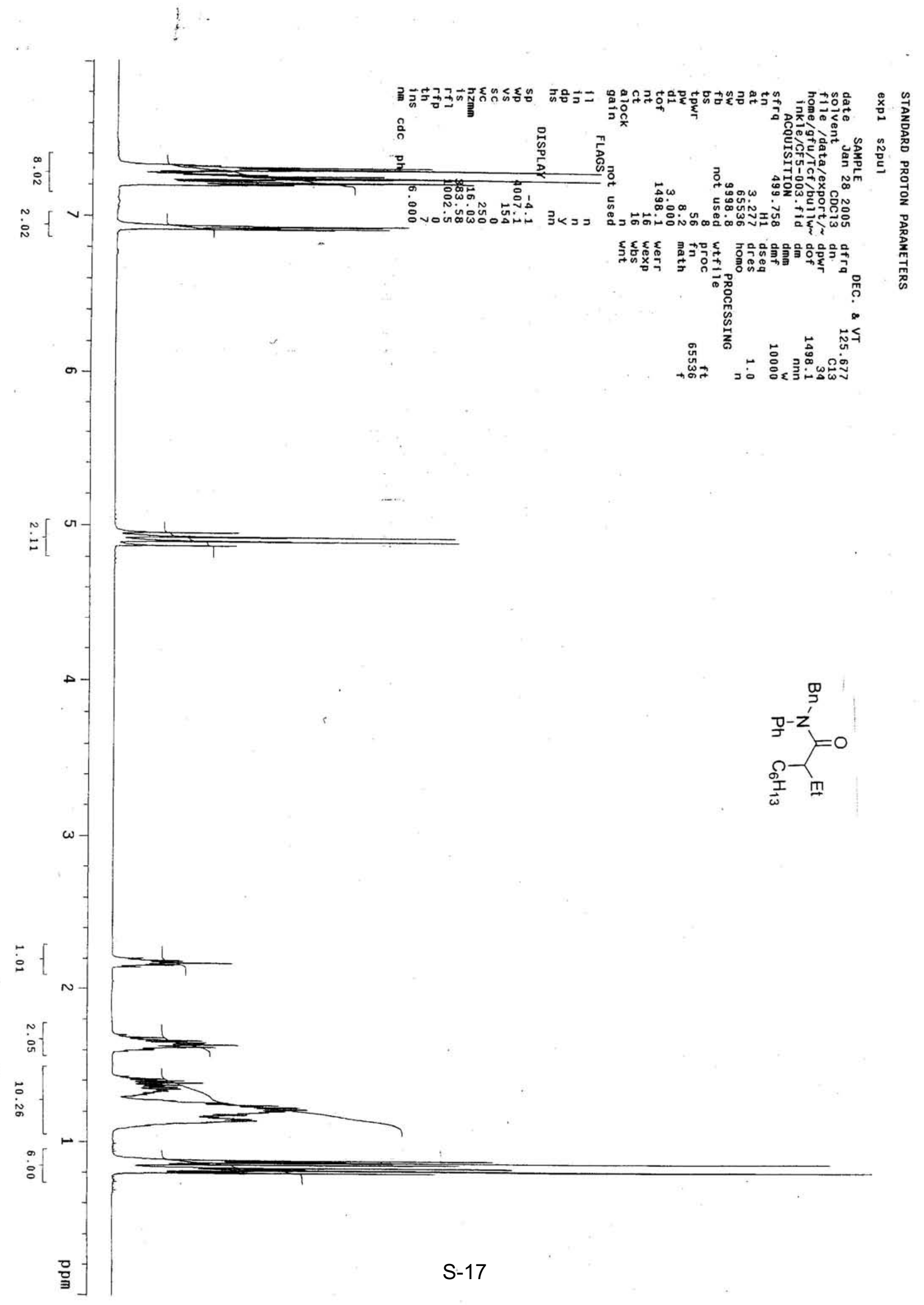




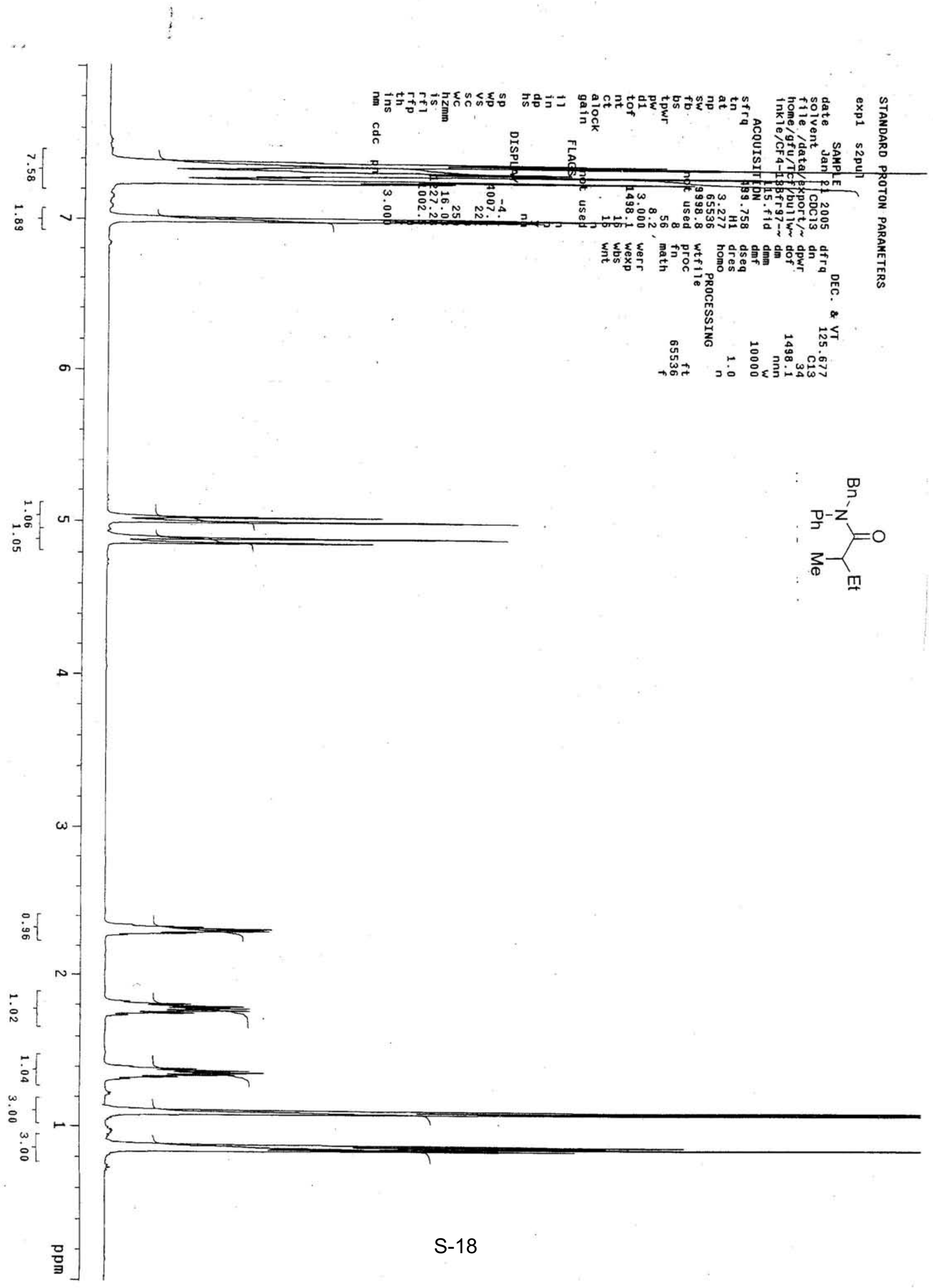




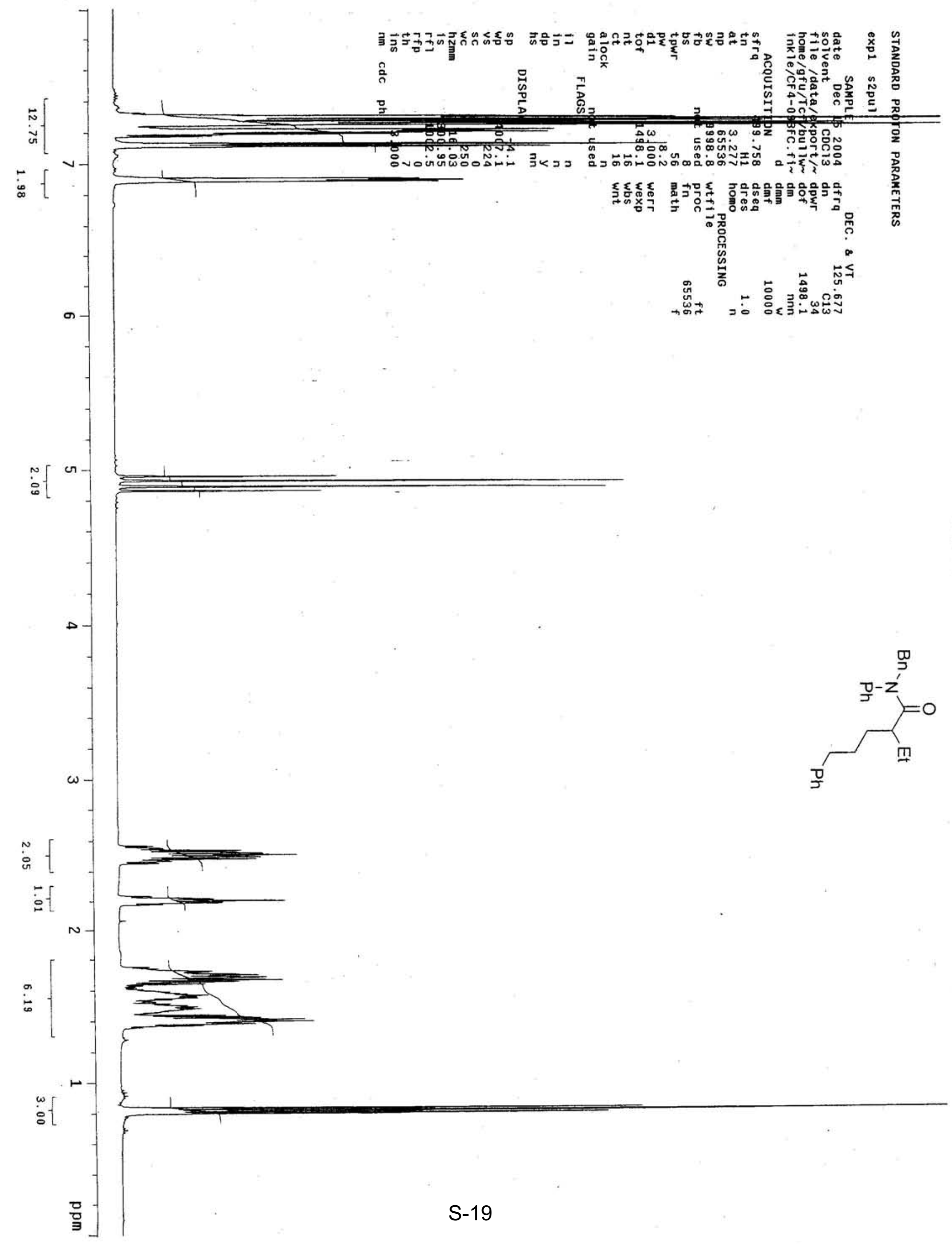




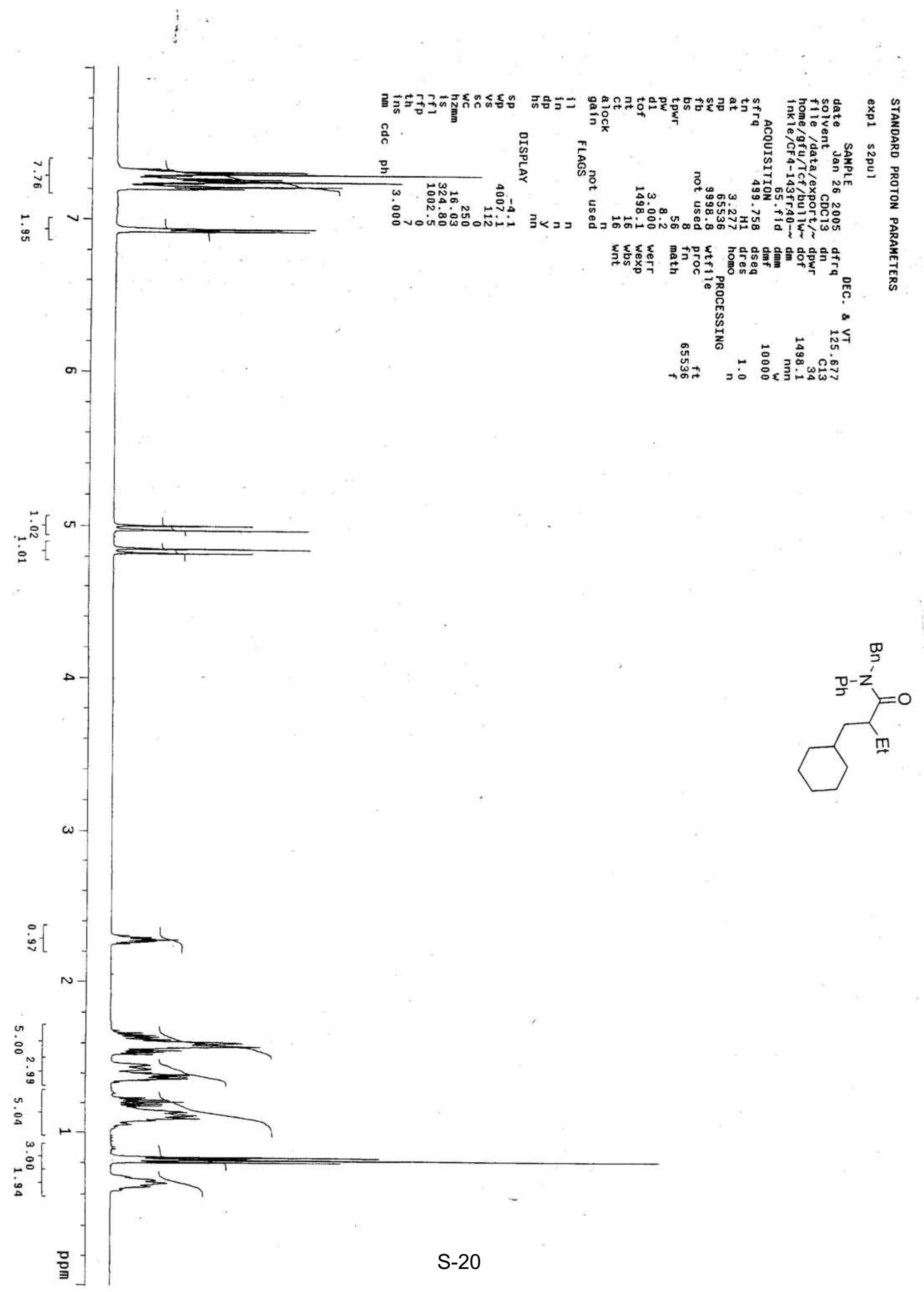




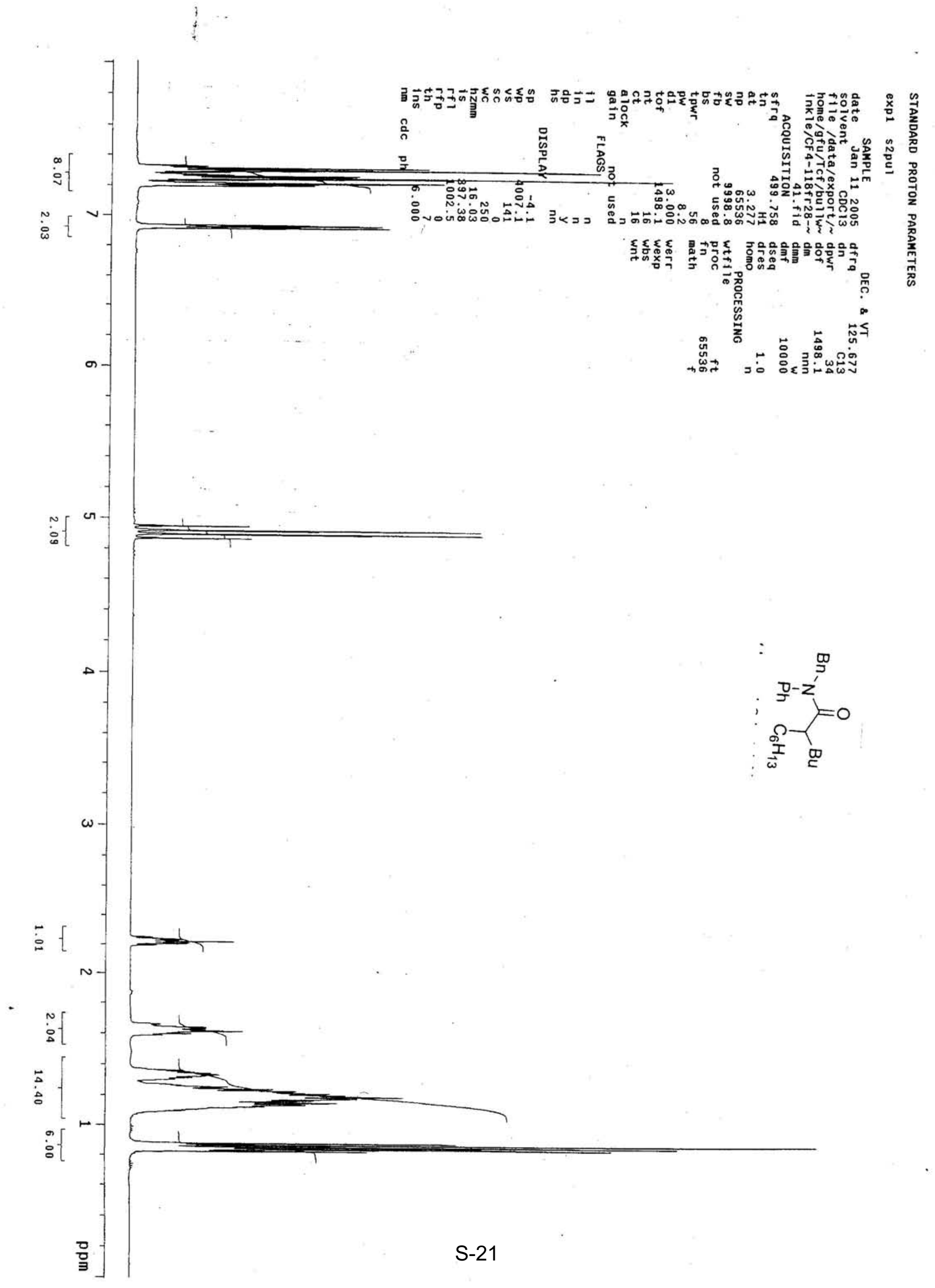




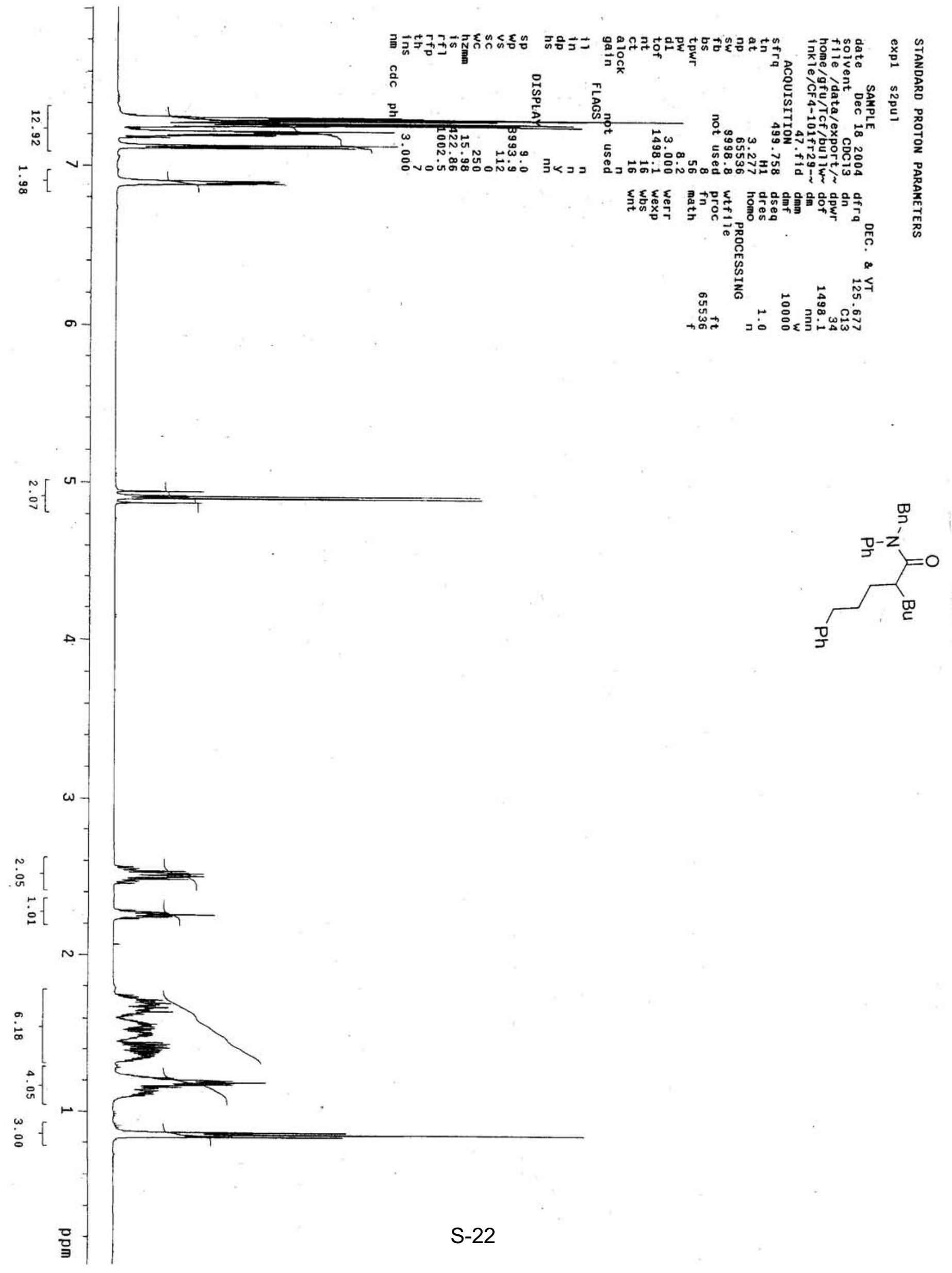




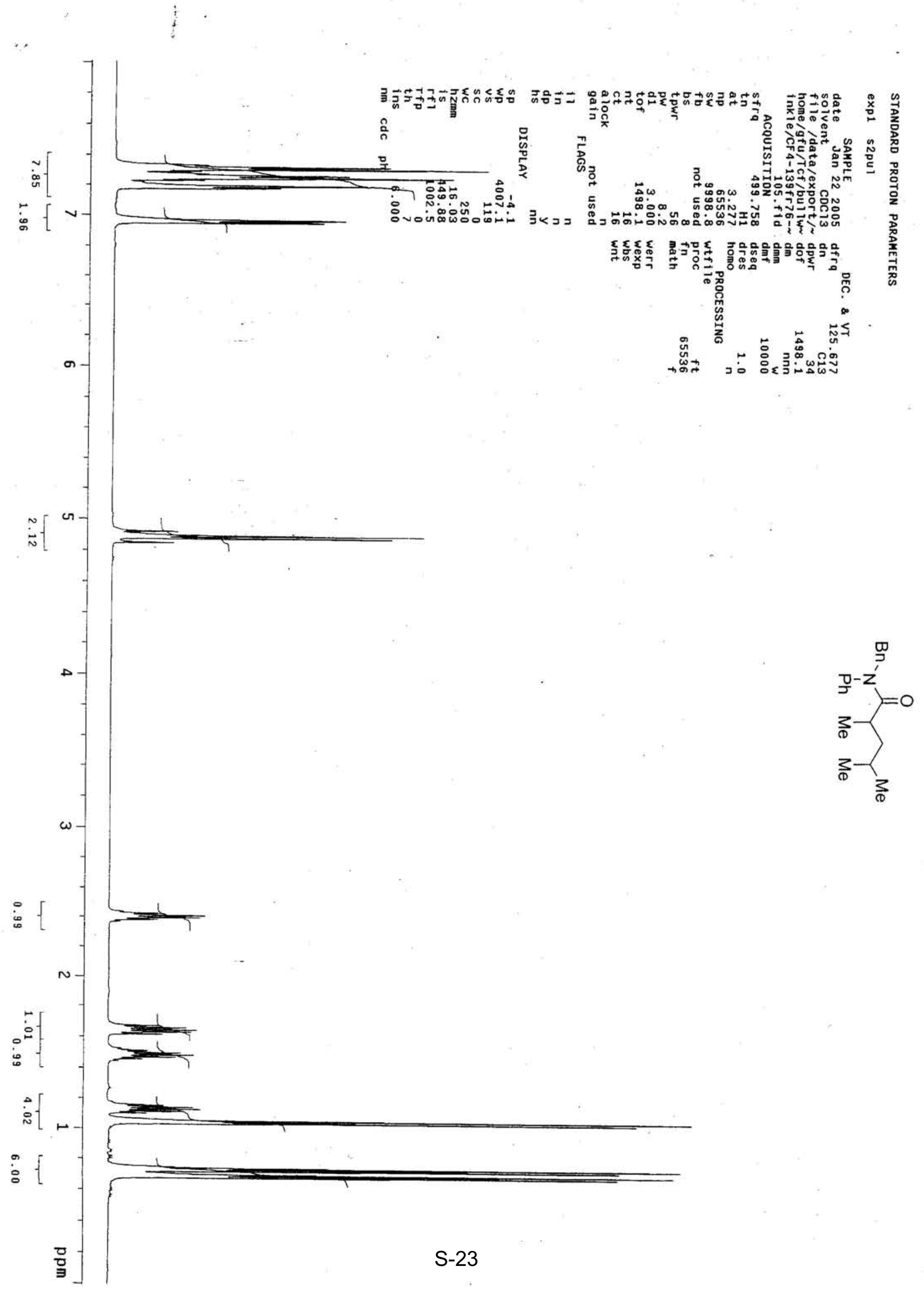




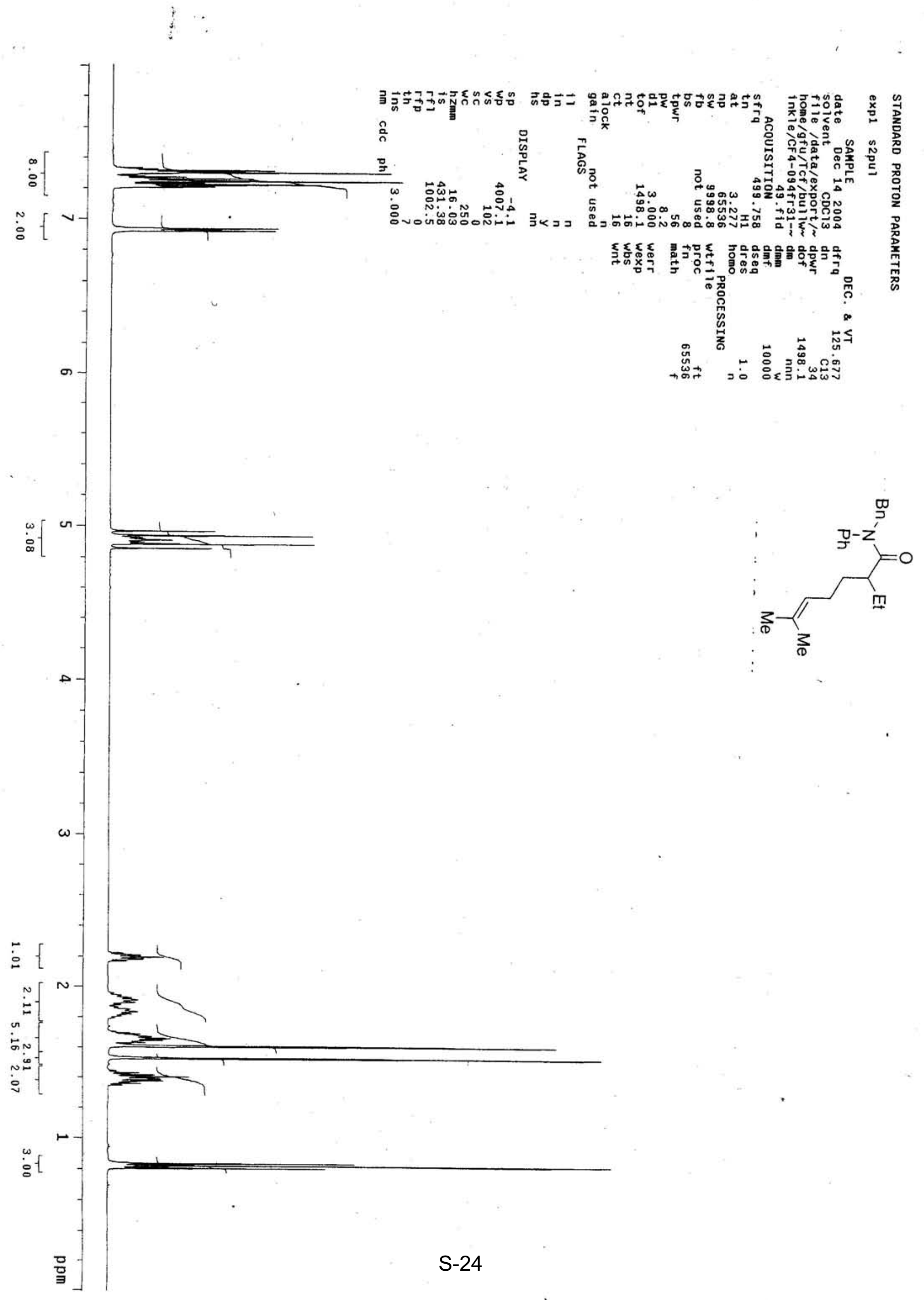




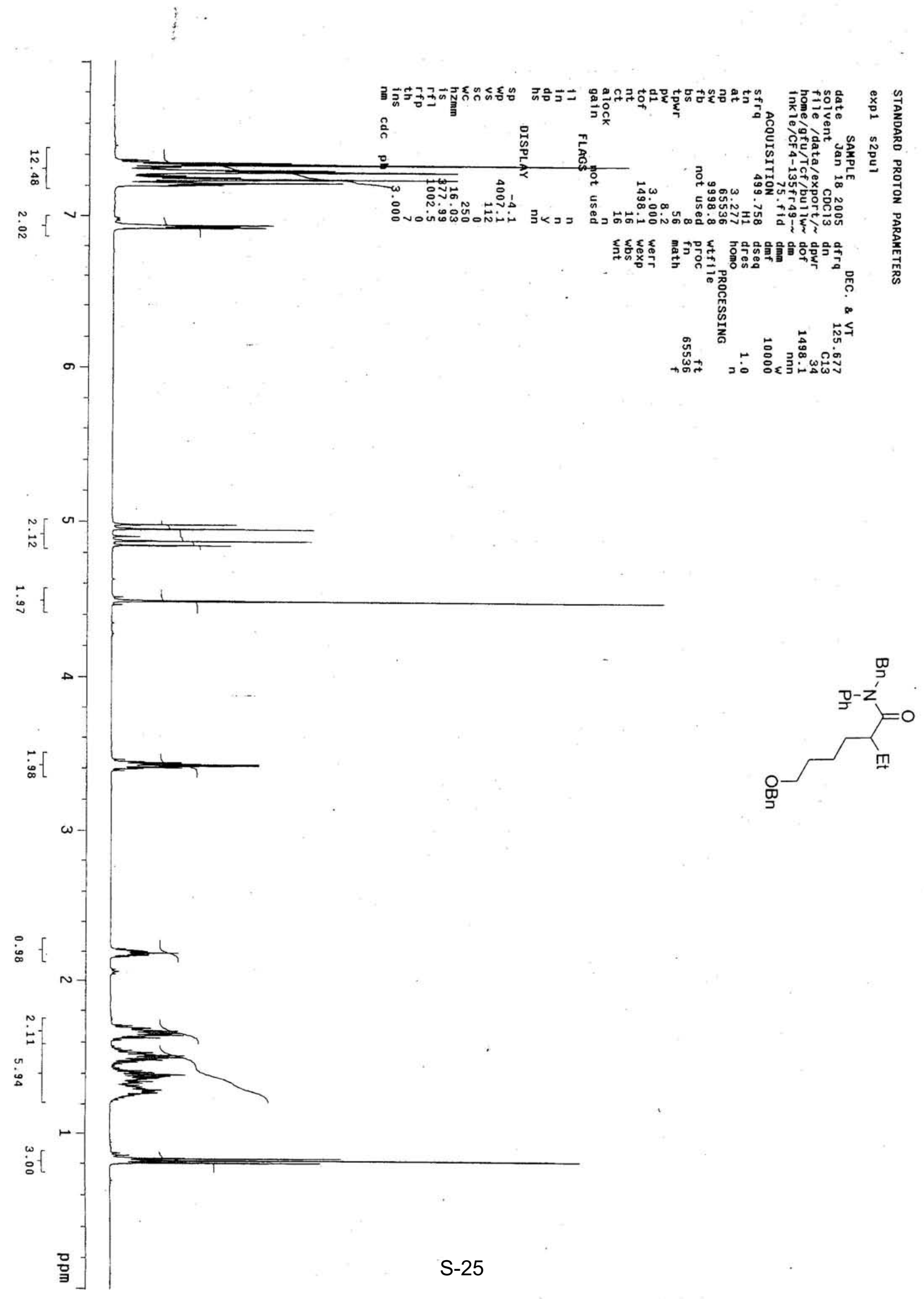




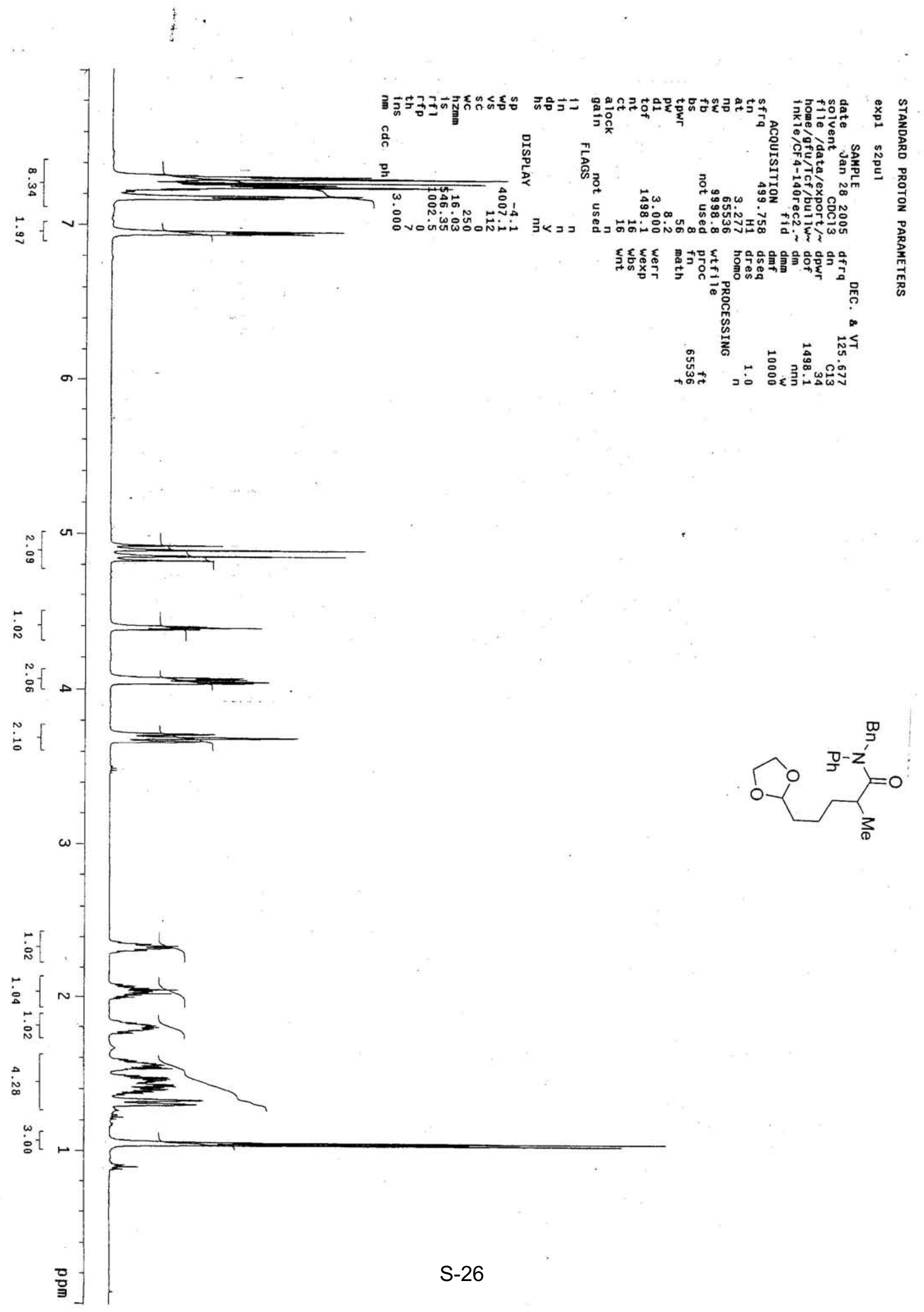




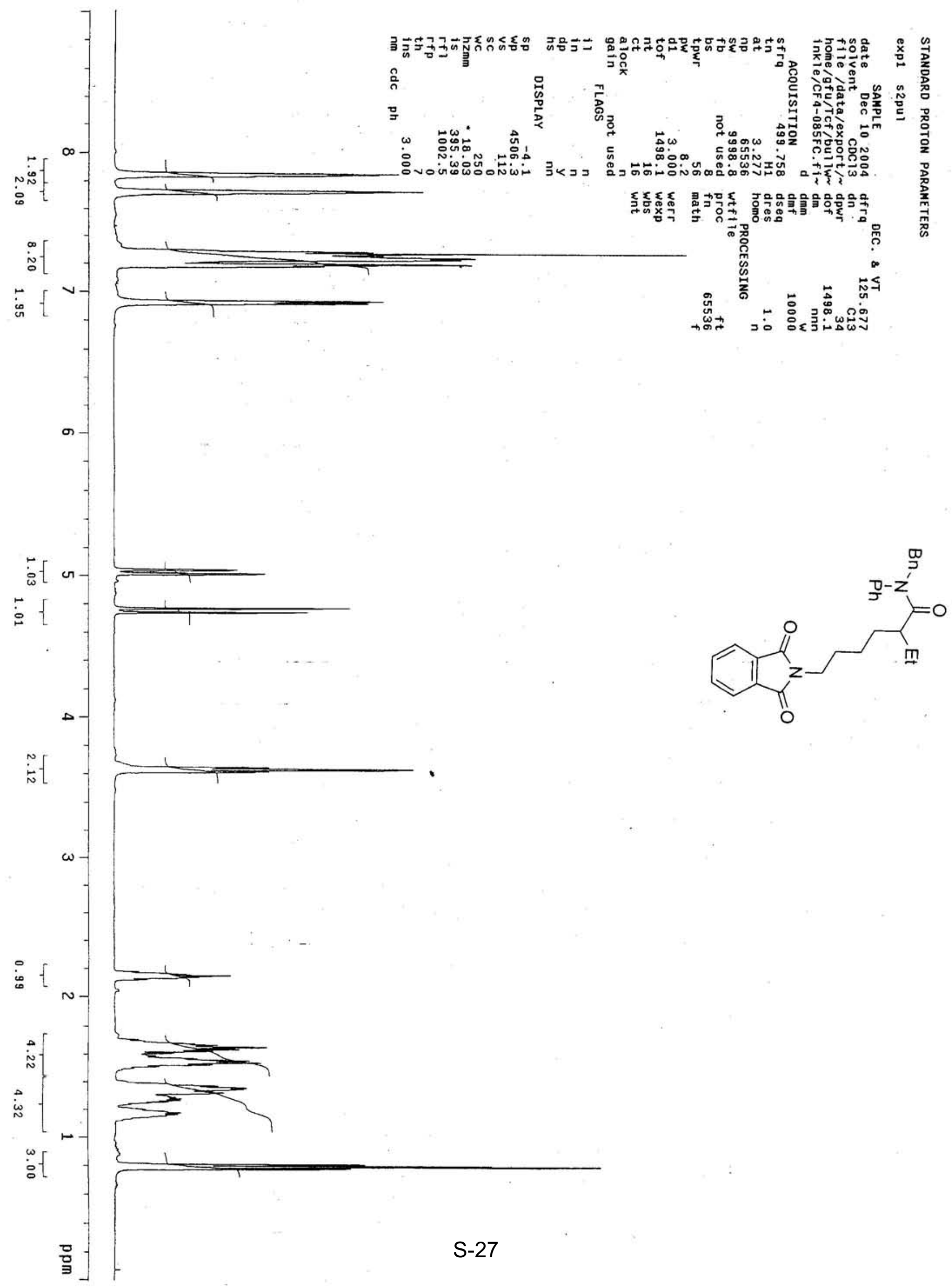




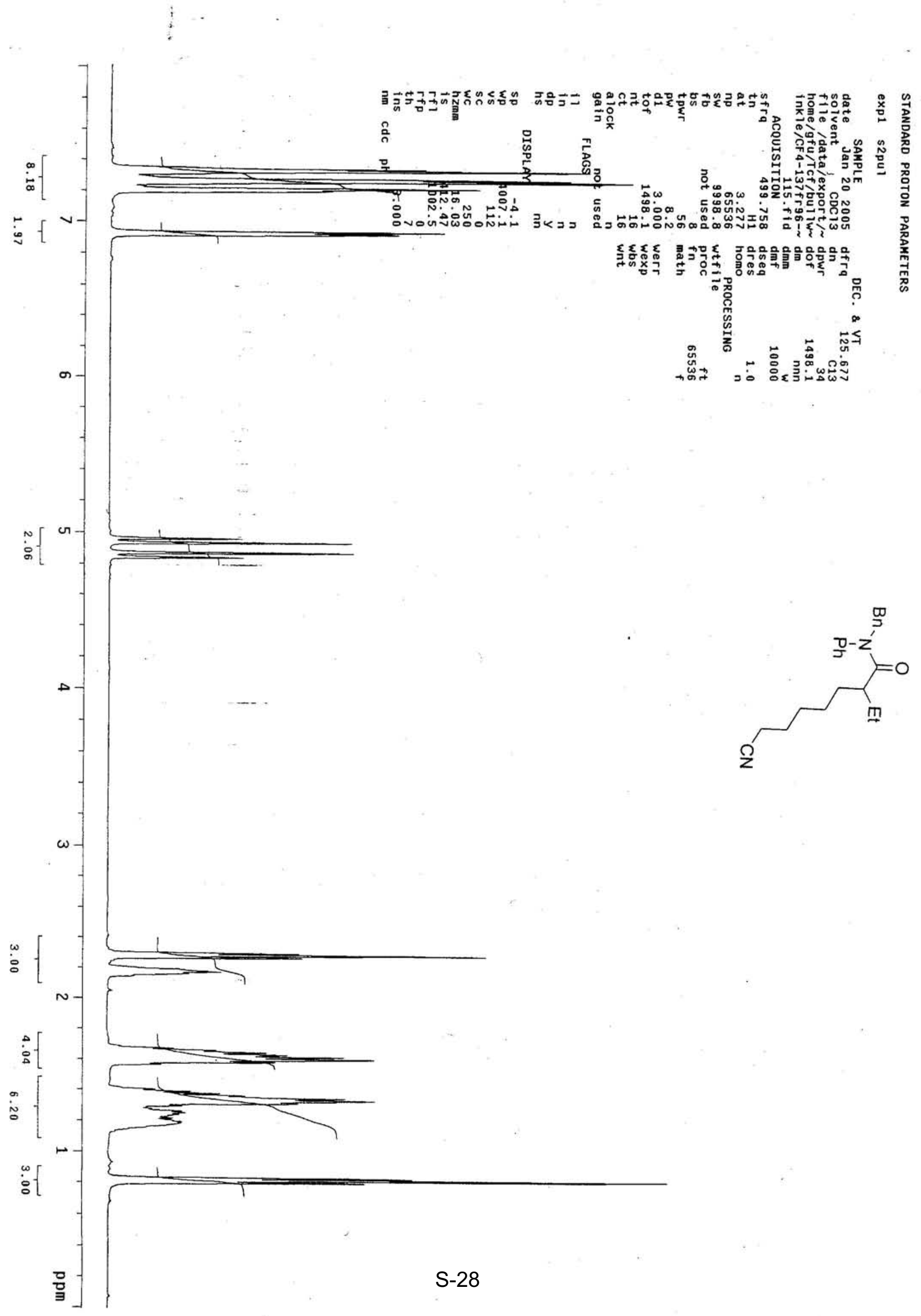




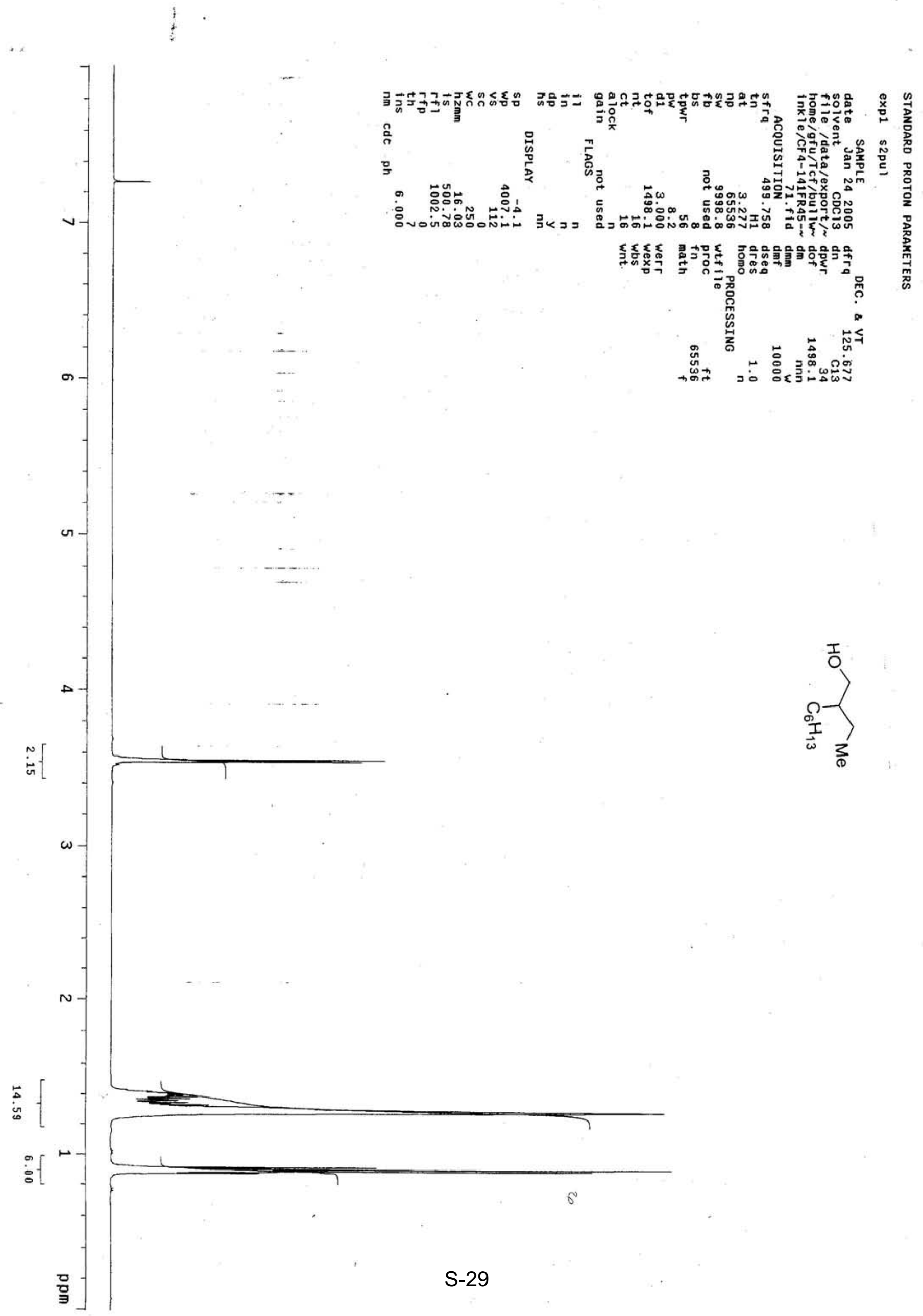

